\title{
The effects of protein crowders on small molecule drug diffusion
}

Debabrata Dey ${ }^{1}$, Ariane Nunes-Alves ${ }^{2,3,5}$, Rebecca C Wade ${ }^{2,3,4}$ and Gideon Schreiber ${ }^{1}$

${ }^{1}$ Department of Biomolecular Sciences, Weizmann Institute of Science, Israel

${ }^{2}$ Molecular and Cellular Modeling Group, Heidelberg Institute for Theoretical Studies, Schloss-

Wolfsbrunnenweg 35, 69118 Heidelberg, Germany

${ }^{3}$ Center for Molecular Biology (ZMBH), DKFZ-ZMBH Alliance, Heidelberg University, Im

Neuenheimer Feld 282, 69120 Heidelberg, Germany

${ }^{4}$ Interdisciplinary Center for Scientific Computing (IWR), Heidelberg University, Im

Neuenheimer Feld 205, Heidelberg, Germany.

${ }^{5}$ Current address: Institute of Biotechnology, Technische Universität Berlin, 13355 Berlin,

Germany

Debabrata Dey and Ariane Nunes-Alves contributed equally to this work.

Corresponding authors:

gideon.schreiber@weizmann.ac.il

rebecca.wade@h-its.org 


\begin{abstract}
Crowded environments affect the pharmacokinetics of drug molecules. Here, we investigate how three macromolecular protein crowders, bovine serum albumin, hen eggwhite lysozyme and myoglobin, influence the translational diffusion rates and interactions of four low molecular-weight drugs, fluorescein, doxorubicin, glycogen synthase kinase-3 inhibitor SB216763 and quinacrine. Using Fluorescence Recovery After Photo-bleaching in Line mode (Line FRAP), Brownian dynamics simulations and molecular docking, we find that the diffusive behavior of the small molecules is highly affected by selfaggregation, interactions with the proteins, and surface adhesion. Fluorescein diffusion is decreased by protein crowders due to their interactions. On the other hand, for doxorubicin, the presence of protein crowders increases diffusion by reducing surface interactions. SB216763 shows a third scenario, where BSA, but not myoglobin or lysozyme, reduces self-aggregation, resulting in faster diffusion. Quinacrine was the only small molecule whose diffusion was not affected by the presence of protein crowders. The mechanistic insights gained here into the effects of interactions of small molecules with proteins and surfaces on the translational diffusion of small molecules can assist in optimizing the design of compounds for higher mobility and lower occlusion in complex macromolecular environments.
\end{abstract}

\title{
Significance statement
}

The activity of small molecules is directly related to their active concentration. This, in turn, relates to their molar concentration and their activity coefficient. Any deviations from ideal behavior affects the small molecule activity. This is particularly important for drugs, where we seek to optimize their molar activity. Here, we follow the diffusional activity of four small molecule drugs in various solutions, with and without protein crowders. The study, which involves experimental measurements and theoretical simulations, shows that 3 of the 4 drugs do not diffuse normally, either due to aggregation, protein binding or surface adhesion. We show the mechanisms driving the abnormal diffusion, and how it can be reduced. Lessons learned from this study can be implemented into drug design.

\section{Introduction:}


Most drugs have molecular weight $<1 \mathrm{kDa}$ and an octanol/water partition $(\log \mathrm{P})$ of $1-6$ (1). For a drug to be orally absorbed, it must be hydrophobic to partition into the lipid bilayer, but not to an extent that will result in permanent absorbance into the bilayer (1). Overall, this results in drugs that self-associate as well as binding to hydrophobic cellular components through soft interactions (2), which in turn decrease their active concentration. In addition to self-aggregation in polar aqueous/buffer-like solvents, small molecule drugs tend to interact with or absorb to glass or plastic surfaces, further complicating diffusion measurements (3). Overlooking these factors may lead to spurious observations that can negatively impact studies of small molecule drugs (4-7). Moreover, while biophysical characterization of these drugs is done in vitro, they are required to be active in a complex, crowded environment, containing membranes and macromolecules at concentrations of up to $300 \mathrm{mg} / \mathrm{mL}(8)$.

For small molecules to reach their target within a crowded milieu, they have to freely diffuse and avoid off-target interactions (9). Passive diffusion is considered to be a primary mechanism of intracellular drug transport (10). However, more recently carriermediated transport $(10,11)$ or a combination of the two were also identified (12). The study of the diffusion of small organic molecules inside complex environments is therefore highly relevant for drug design $(9,13)$, as well as for soft matter and biochemical (14) studies more generally (15). The challenge (16) is that most small molecules are not fluorescent in the visible spectrum, making it almost impossible to follow them by optical methods (17). Conversely, proteins can usually be fluorescently labeled without significant perturbation of their diffusion rate and function, making it relatively easy to study their diffusion (18).

Here, we investigated the diffusion of three fluorescent organic small molecule therapeutic drugs and one diagnostic marker in solutions containing proteins as crowders. Doxorubicin (DOX) is widely used as an anti-cancer agent (19, 20); quinacrine dihydrochloride has been used for antimalarial and antiprotozoal therapy $(21,22)$; glycogen synthase kinase-3 (GSK3) inhibitor SB 216763 is a potent selective and ATPcompetitive inhibitor of glycogen synthase kinase-3 and significantly prevents lung inflammation and fibrosis in mouse models (23-25). DOX and quinacrine behave as weak bases at physiological $\mathrm{pH}$ (26). Unlike the three therapeutic drugs, fluorescein in its salt 
form (which is widely used as a biological marker in angiographic assays) is a negatively charged molecule at physiological $\mathrm{pH}(27,28)$. The four molecules investigated are structurally and chemically different from each other (Figure 1). They were chosen according to their spectral properties, with fluorescence in the visible spectrum being a prerequisite for their application in this study (Figure 1). The diffusion and aggregation of these low MW molecules were evaluated in buffer, and with bovine serum albumin (BSA), hen egg-white lysozyme (HEWL), and myoglobin as molecular crowders $(29,30)$. Selfaggregation and binding to these proteins can potentially mimic their behaviors in body fluids or inside the cell $(31,32)$. Diffusion rate measurements provide a good proxy for estimating these protein-ligand associations or their aggregation state in diversified environments.

A wide range of computational and experimental techniques have been developed to compute and measure diffusion rates, as well as to study molecular association. On the computational side, Brownian dynamics (BD) (33) and molecular dynamics (MD) (16, 30, 34-36) simulations are widely used. In both methods, the system can be considered in atomic detail, but in BD simulations, the molecules are generally treated as rigid bodies and an implicit solvation model is used. These simplifications allow BD simulations to routinely achieve tens of microseconds of simulation time with systems containing hundreds of solute molecules. BD simulations have been previously employed to compute diffusional properties of proteins, revealing, for example, molecular details about the reduction in diffusion rates of BSA, myoglobin, hemoglobin and $\gamma$-globulin under crowding conditions $(37,38)$ or the adsorption of HEWL to an inorganic surface $(39,40)$.

Among the experimental techniques, fluorescence recovery after photobleaching (FRAP) (41), fluorescence correlation microscopy (FCS) (42), and single-particle tracking (SPT) (43) are the most popular. Each method has its benefits and limitations (44). FCS is considered the gold standard for this purpose; however, its application can be challenging. For one, it requires high quantum yield (which is rare for drugs), second it can be performed only at very low concentrations, which may be much lower compared to that found in vivo. Therefore, FRAP is the technique most widely used by experimental biologists (44). It is fast, non-invasive, highly specific, and relatively easy to perform (44). Moreover, FRAP 
can be used on molecules with poor quantum yields and at high, biologically relevant concentrations (41).

We previously developed the Fluorescence Recovery After Photo-bleaching in Line mode (Line FRAP) method to monitor the diffusion rates of proteins in various environments (45). The main advantage of Line-FRAP over conventional FRAP is the much faster data acquisition rate, which allows measurements for fast diffusing molecules. The apparent diffusion coefficients calculated from FRAP measurements are $\mathrm{D}_{\text {confocal }}(45)$, which are calculated according to equation 1:

$D_{\text {confocal }}=\frac{r_{e}^{2}+r_{n}^{2}}{8 \tau_{1 / 2}} \quad$ eq. 1

where $\tau_{1 / 2}$ is the half-time of recovery and $r_{e}$ and $r_{n}$ are the effective and nominal bleach

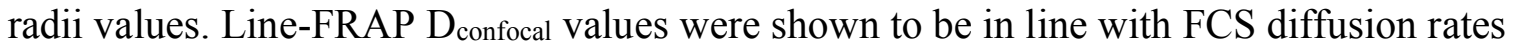
for proteins (45). However, for small molecules, they provide only relative 3D diffusion coefficients (46). An alternative way to present FRAP results is by reporting only $\tau_{1 / 2}$ values. However, as seen from eq. 1, this completely ignores the contribution from the bleach radius (which is squared) $(47,48)$. In particular, $r_{e}$ varies, depending on the type of molecule and the diffusion condition, making its measurement critical to obtain reliable estimates of diffusion rates.

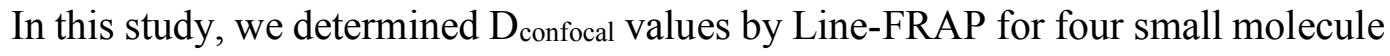
drugs using three different proteins as crowders. The derived diffusion coefficients are shown to depend on the self-aggregation properties of the small molecules, their binding to proteins and surfaces, and the buffer solution conditions. The protein crowders affect the solubility and diffusion rates of the drugs in a manner that is dependent on the properties of the different protein crowders used. Complementary steady-state fluorescence quenching and size exclusion chromatography experiments provide thermodynamic data that reveal differences in the associations between the drug molecules and the protein crowders. Furthermore, Brownian dynamics simulations and molecular docking for the systems studied experimentally shed light on the intermolecular interactions and molecular mechanisms responsible for the differences in the diffusion coefficients measured for the different small molecule and protein crowder combinations.

\section{Results}




\section{Fluorescein disodium salt}

Fluorescein is a negatively charged organic small molecule (MW = 376.3 Da, Figure 1A). As per our stepwise experimental design, we initially measured diffusion rates of fluorescein in PBS buffer with and without protein crowders. Figure 2A shows the calculated diffusion rates in DMSO, PBS and Tween20 and Figure 2B shows micrograph

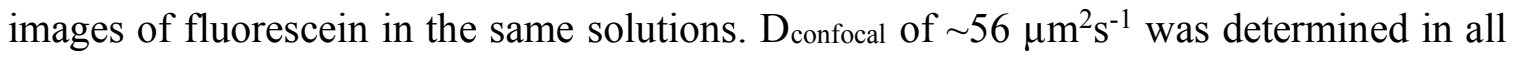
these cases, with no evidence for aggregation of fluorescein. Next, we measured fluorescein's diffusion in the presence of increasing concentrations of BSA, HEWL, and myoglobin (Figure $2 \mathrm{C}$-E). Here, we were surprised to see that, even at low concentrations of protein, the presence of BSA, HEWL and myoglobin slowed down diffusion of fluorescein significantly (Figure 2E). Since the fraction of excluded volume due to the presence of protein crowders is low at concentrations below $20 \mathrm{mg} / \mathrm{mL}$, we conclude that quinary or weak interactions between fluorescein and the protein crowders are the main drivers of the reduction in the diffusion rates of fluorescein, in line with previous publications (49). HEWL had the biggest effect on diffusion, followed by BSA and myoglobin. Interestingly, for myoglobin, increasing concentrations above $5 \mathrm{mg} / \mathrm{mL}$ resulted in an increase in diffusion rates, which returned to their level without crowder at $50 \mathrm{mg} / \mathrm{mL}$ (Figure 2E). Micrographs in the presence or absence of protein crowders did not show aggregation (Figure 2B and D) and FRAP fully recovered after bleach at all conditions (Figure 2C).

To investigate the factors influencing the diffusion of fluorescein, BD simulations were performed for fluorescein and the same concentrations of protein crowders as present in FRAP experiments. The results show that the effects of the crowders modelled in these simulations (excluded volume, electrostatic and hydrophobic interactions between rigid solutes) result in modest reductions of up to $15 \%$ in the computed translational diffusion coefficients of the small molecules at crowder concentrations up to $50 \mathrm{mg} / \mathrm{mL}$ (Figure S1). The translational diffusion coefficients for fluorescein computed from BD simulations did not show the markedly large decrease observed in the experiments. Thus, it can be deduced that other factors, not included in the BD simulation model, are important for the experimentally observed reduced diffusion upon addition of protein crowder. Such factors may include surface interactions (see below) and molecular aggregation, that relies on 
conformational variability and induced fit, which are neglected in the rigid-body BD simulations, as well as inaccuracies in the force field used.

Nevertheless, the BD simulations revealed differences in the interactions of fluorescein with the three protein crowders which were examined by computing the number of intermolecular contact interactions. Contacts were defined as present if nonhydrogen atoms (at least one in a protein crowder and at least one in a small molecule) were within $4.5 \AA$ of each other. This distance was chosen to capture electrostatic and van der Waals interactions between protein crowders and fluorescein. The number of proteinfluorescein contact interactions (Figures $3 \mathrm{~A}$ ) and the peak of the radial distribution function (RDF) for protein-small molecule distances (Figures 3B) are higher in the presence of HEWL, showing that the interactions of fluorescein are stronger with this protein crowder. The stronger interactions between fluorescein and HEWL are consistent with the experimentally observed stronger reduction in the diffusion coefficient of fluorescein in the presence of HEWL than in the presence of the other two protein crowders. The stronger interaction between fluorescein and HEWL can be explained by the strong electrostatic interaction between them, since at the $\mathrm{pH}$ of the experiments (7.4), fluorescein is negatively charged ( -2 e) and HEWL is positively charged (+8 e). Another factor that contributes to this strong electrostatic interaction is the distribution of the molecular electrostatic potential of HEWL, which has a large positively charged region on the surface that interacts mostly with fluorescein during the BD simulations (Figure S5). Despite their negative net charges, myoglobin (-2 e) and BSA (-16 e) can also make favorable electrostatic interactions with fluorescein through regions of positive electrostatic potential on their surfaces and these are the parts of the protein surface with the greatest occupation of contacts with fluorescein during the $\mathrm{BD}$ simulations (Figure S5). However, these interactions are weaker than for HEWL and result in fewer contacts and much less pronounced peaks in the RDF (Figures $3, \mathrm{~S} 3$ and S4).

To quantify the protein-small molecule binding affinities, steady-state fluorescence quenching experiments were done. Association of the small molecules with the proteins causes a change in the environment around buried tryptophan residues (responsible for intrinsic fluorescence properties of proteins), which results in the quenching of fluorescent signals from the protein (50). For example, BSA contains two tryptophan residues, Trp- 
134 and Trp-212, located in the first and second domains of hydrophobic protein regions (50). The decrease of fluorescence intensity for BSA was monitored at $344 \mathrm{~nm}$ wavelength for the drug-protein pairs. Figures S6 A-C show representative fluorescence quench spectra for fluorescein with BSA, HEWL, and myoglobin. We assume that the observed changes in the fluorescence are a result of the interaction between the small molecule and the protein. Therefore, corresponding plots of $\left(\mathrm{F}_{0}-\mathrm{F}\right) /\left(\mathrm{F}_{0}-\mathrm{F}_{\mathrm{c}}\right)$ versus fluorescein concentration [Q] in molarity (Figure 2F) were used to determine the affinity between them, using equation 2 (see Materials and methods). BSA, HEWL, and myoglobin bind fluorescein with affinities of $1.7 \pm 0.2 \mu \mathrm{M}, 3.8 \pm 0.6 \mu \mathrm{M}$ and $3.0 \pm 0.5 \mu \mathrm{M}$, respectively (Figure $2 \mathrm{~F}$ and Table S1).

Binding of fluorescein to the protein crowders could explain its reduced diffusion in their presence. To explore this further, we first measured $\mathrm{D}_{\text {confocal }}$ of BSA (labelled with CF514 dye) at concentrations between $5-50 \mathrm{mg} / \mathrm{mL}$. Indeed, BSA diffusion is slowed at higher concentrations, probably due to self-crowding (Figure 2G). However, diffusion is reduced to a smaller extent than that measured with the addition of fluorescein to BSA. To understand why BSA + fluorescein diffuses slower, we measured $\mathrm{D}_{\text {confocal }}$ of fluorescein and labelled BSA (with CF514 Dye) separately and simultaneously, using excitation at either $440 \mathrm{~nm}$ (fluorescein) or $515 \mathrm{~nm}$ (CF514 dye). We note that fluorescein and CF514 dye have an overlap in their excitation/emission spectra. However, comparing the percentages of bleach values (Figure S7) for the different species alone or together, shows that also in the BSA/fluorescein mixture, the percent bleach of BSA or BSA and fluorescein, when excited at $515 \mathrm{~nm}$ is $\sim 65 \%$, while for fluorescein (with or without BSA, excited at $440 \mathrm{~nm})$, it is $80-85 \%$. Thus, excitation at $440 \mathrm{~nm}$ measures mostly fluorescein diffusion, whereas excitation at $515 \mathrm{~nm}$ measures mostly BSA diffusion. Figure 2G clearly shows that the diffusion rate of labelled BSA $(515 \mathrm{~nm})$ in the presence of fluorescein (440 $\mathrm{nm}$ ) is slower than the diffusion rate of BSA alone (Figure $2 \mathrm{G}$ ), and that it is very similar to the diffusion rate of fluorescein in the presence of BSA. The slower diffusion rates of BSA could be due to a higher oligomerization state caused by the presence of fluorescein. Oligomerization would lead to an increase in the Stokes radius, resulting in slower diffusion rates. Only a modest reduction in the diffusion coefficient of BSA in the presence of fluorescein with increasing BSA concentration up to $50 \mathrm{mg} / \mathrm{mL}$ was observed in BD 
simulations (Figure S2), again indicating that more extensive aggregation of $\mathrm{BSA}+$ fluorescein is present than that captured by the BD rigid body model and force field.

\section{Doxorubicin:}

The chemical structure and spectroscopic properties of DOX (MW=580 Da) are shown in

Figure 1B. It diffuses surprisingly slowly in PBS buffer, with $D_{\text {confocal }} \sim 12.6 \mu \mathrm{m}^{2} \mathrm{~s}^{-1}$ (Figure 4A), while. the infinite dilution translational diffusion coefficient of DOX (Stokes radius of $5.1 \AA$ ), $D_{\text {trans, }}$ is calculated to be $412 \mu \mathrm{m}^{2} \mathrm{~s}^{-1}$ (Table S2). Next, we measured the diffusion of DOX in DMSO, where $\mathrm{D}_{\text {confocal }}$ increased to $47 \mu \mathrm{m}^{2} \mathrm{~s}^{-1}$ (Figure 4A). Addition of 0.1 percent of the Tween 20 surfactant, which promotes the solubilization of aggregated hydrophobic DOX molecules and prevents surface attachment (3), increased diffusion in PBS to $27 \mu \mathrm{m}^{2} \mathrm{~s}^{-1}$, with a similar rate measured with $10 \mathrm{mg} / \mathrm{mL}$ of BSA, with or without Tween 20 (Figure 4A). Micrographs of DOX in different solutions (Figure 4A, right panel) do not show obvious high MW aggregates. However, this does not exclude DOX from forming lower MW aggregates, as has been suggested previously (51), and could explain the slow diffusion of DOX in PBS. To further verify the $\mathrm{D}_{\text {confocal }}$ values, we measured them after 10 and $63 \mathrm{~ms}$ bleach times. The corresponding recovery curves and bleach radius are shown in Figure S8. The data clearly show much slower recovery curves after $63 \mathrm{~ms}$ bleach, which are accompanied by a wider bleach radius. However, the $\mathrm{D}_{\text {confocal values }}$ calculated from $\tau_{1 / 2}$ and $r_{e}$ were similar for the different bleach times (Table S3), in line with what we have previously shown for protein diffusion (45).

Next, we measured DOX diffusion after addition of different concentrations of

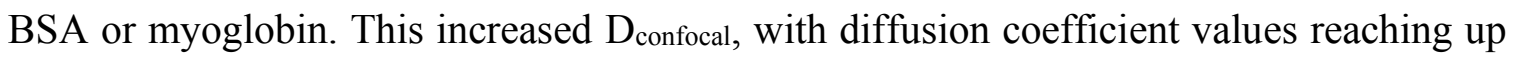
to $\sim 40 \mu \mathrm{m}^{2} \mathrm{~s}^{-1}$ (Figures $4 \mathrm{C}, \mathrm{D}$ ), which are close to the value recorded in DMSO (Figure 4A). Titration of increasing concentrations of BSA, from $0.01-200 \mathrm{mg} / \mathrm{mL}$, showed that the maximum diffusion coefficient is reached at $10-20 \mathrm{mg} / \mathrm{mL}$, with a decrease observed at higher BSA concentrations. This slow down can be associated with the excluded volume effect, which starts to be significant for BSA at 50 and $200 \mathrm{mg} / \mathrm{mL}$ (fraction of occupied volume of 0.035 and 0.14 , respectively). BD simulations (which include intermolecular forces as well as excluded volume effects) show that not only the diffusion rate of 
doxorubicin, but also that of BSA itself, is reduced as BSA concentrations increase from 20 to $200 \mathrm{mg} / \mathrm{mL}$ (Figures S1 and S2), in agreement with the experimental results.

Next, we determined the binding of DOX to BSA and myoglobin by fluorescence quenching titration experiments (Figures 4F and G \& S6 D and E). BSA and myoglobin bind DOX with low affinities of $16.5 \pm 2.5 \mu \mathrm{M}$ and $18.4 \pm 3.1 \mu \mathrm{M}$, respectively (Figure $4 \mathrm{~F}$ and $\mathrm{G}$ and Table $\mathrm{S} 1$ ), in agreement with a previous study (50).

To investigate whether slow diffusion of DOX in PBS is a result of surface attachment or self-aggregation, we determined the fluorescence intensity as a function of the distance from a glass surface for DOX and performed the same measurements for fluorescein and quinacrine for comparison. Whereas for fluorescein, we saw a homogeneous distribution of the small molecule in the drop, for DOX, surface attachment was clearly observed (Figures $5 \mathrm{~A}$ and B). This is in line with a previous report that DOX can adsorb to a polypropylene surface (3). We then used BSA, myoglobin and PEG8000 solutions to coat the glass surface and thereby decrease the surface attachment and adsorption of the DOX molecules. Out of the three, myoglobin acted as the best surface coating agent for DOX molecules (Figure 5B), resulting in almost complete removal of DOX from the surface. In addition, myoglobin coating of the glass surface resulted in a large increase in $\mathrm{D}_{\text {confocal }}$ compared to DOX in PBS solution (Figure 5E), giving a value of $36 \mu \mathrm{m}^{2} \mathrm{~s}^{-1}$, which is similar to the maximum value measured in the presence of BSA. The effect of BSA or PEG8000 coating of the glass surface on the $\mathrm{D}_{\text {confocal values was minimal }}$ and DOX remained attached to the glass surface even after coating (Figure 5B and E). BD simulations in the absence of a surface show a low number of DOX-protein contacts (Figure S3) and small, broad RDF peaks for DOX-protein distances (Figure S4). This agrees with the experimental results, which show that the main effect of the protein crowders, especially at low concentrations, is prevention of DOX attachment to the glass surface, rather than slowed diffusion by quinary interactions with protein crowders, as observed for fluorescein. Nonetheless, it should be noted that quinary interactions between DOX and BSA are expected, as reported in a previous study (50).

BD simulations performed with a silica surface to mimic the glass and DOX molecules or the protein crowders at a concentration of $50 \mathrm{mg} / \mathrm{mL}$ show that all these types of molecules can adsorb to the negatively charged silica surface (Figures 5G and S9), in 
agreement with previous studies using simulations or experiments $(39,52-54)$. However, a higher number of myoglobin and HEWL molecules adsorbed to the surface compared to DOX and BSA, indicating that myoglobin and HEWL interact better with the surface and may be better at preventing DOX surface attachment, consistent with the experimental results observed for myoglobin and BSA. Measuring diffusion of DOX in PBS solution

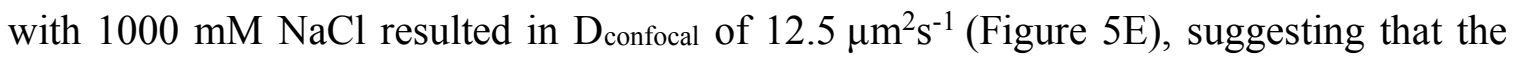
electrostatic attraction of the positively charged DOX to the negatively charged glass surface is not required for surface adsorption which is instead driven by hydrophobic interactions, in line with a previous report of DOX adsorption to polypropylene surfaces (3).

\section{GSK3 inhibitor SB216763}

GSK-3 is a protein kinase that is active in a number of central intracellular signaling pathways, including cellular proliferation, migration, glucose regulation, and apoptosis (23-25). The GSK-3 inhibitor SB216763 is currently being evaluated for several related malignancies (23-25). The chemical structure and spectroscopic properties of SB216763 are shown in Figure 1C. SB216763 has a strong tendency to aggregate in PBS (Figure 6A). Aggregation is also observed in DMEM media. Of the four small molecules tested, the GSK3 inhibitor is the only one with neutral net charge at the $\mathrm{pH}$ of the experiments. The lack of repulsive electrostatic interactions may facilitate aggregation. Moreover, BD simulations of the GSK3 inhibitor without protein crowders, showed greater selfinteractions than those observed for the other small molecules (Table S4), consistent with the experimental data. The addition of BSA (but not myoglobin or HEWL) eliminates the aggregation, especially at concentrations of 25 and $50 \mathrm{mg} / \mathrm{mL}$. FRAP experiments validate the micrograph observations (Figure 6B to E). Percent FRAP recovery of SB216763 is very low in buffer or in myoglobin, but reaches close to $100 \%$ with the addition of 50 $\mathrm{mg} / \mathrm{mL}$ BSA (see normalized fluorescence in Figure 6B). Also, $\mathrm{D}_{\text {confocal }}$ is much higher in the presence of $50 \mathrm{mg} / \mathrm{mL}$ BSA (Figure 6C). FRAP recovery is in line with the micrographs shown in Figure 6A. Titrating increasing concentrations of BSA into SB216763 solutions

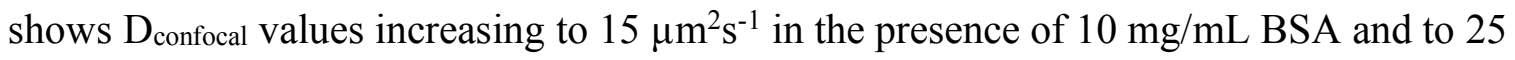
$\mu \mathrm{m}^{2} \mathrm{~s}^{-1}$ in the presence of $100 \mathrm{mg} / \mathrm{mL}$ BSA. This value is similar to the measured $\mathrm{D}_{\text {confocal }}$ 
of fluorescein and BSA at $50 \mathrm{mg} / \mathrm{mL}$ BSA (Figure $2 \mathrm{G}$ ), suggesting that high molecular weight aggregates of GSK3 are completely solubilized. Interestingly, binding experiments of SB216763 to BSA, HEWL, and myoglobin showed affinities of $5.2 \pm 1.2 \mu \mathrm{M}, 1.7 \pm 0.7$ $\mu \mathrm{M}$, and 7.3 $\pm 1.8 \mu \mathrm{M}$ (Figure $6 \mathrm{~F}-\mathrm{H}$, Table S1). Thus, while BSA solubilizes SB216763 much better than the other two proteins, we did not find a relation between solubilization of the small molecule and binding affinity.

Visual inspection of the apo and holo crystal structures of the protein crowders shows that myoglobin does not have a crevice or buried binding site for small molecules larger than molecular oxygen, whereas HEWL and BSA do. Moreover, HEWL and BSA were shown to bind to small molecules and act as potential drug carriers $(50,55,56)$. HEWL can accommodate small molecules in the crevice of its catalytic site, while BSA has four binding cavities that can accommodate small molecules (Figure 6I). Docking of the GSK3 inhibitor to BSA and to HEWL shows that the GSK3 inhibitor can bind to one of the cavities of BSA with a good docking score $(-9.2 \mathrm{kcal} / \mathrm{mol}$, Figure $6 \mathrm{I})$ and to the catalytic site of HEWL with a less favorable docking score $(-7.9 \mathrm{kcal} / \mathrm{mol})$. The preferable deep binding cavities in BSA that can accommodate the GSK3 inhibitor may explain why BSA is the only protein crowder that can reduce the aggregation of this small molecule. The diffusion coefficients computed from BD simulations did not show the trends obtained experimentally for the GSK3 inhibitor in the presence of protein crowders (Figure S1), indicating the importance of factors omitted in the BD model, such as small molecule aggregation and binding of small molecules within rather buried cavities in the protein crowder.

\section{Quinacrine Dihydrochloride:}

Quinacrine dihydrochloride $(\mathrm{MW}=472.9 \mathrm{Da})$, whose chemical structure and spectroscopic properties are shown in Figure 1D, is among the first antimalarial drugs discovered. Recently, it was also suggested to have high potency against SARS-CoV-2 in an in vitro setting $(57,58)$. Quinacrine does not display any significant aggregation issues in buffer solutions (Figure 7 A, B), and it is not absorbed onto glass surfaces (Figure 5C). The addition of BSA, HEWL or myoglobin did not affect its diffusion coefficient ( $D_{\text {confocal }}$ 41- 
$43 \mu^{2} \mathrm{~s}^{-1}$, Figure $\left.7 \mathrm{~A}, \mathrm{C}, \mathrm{D}\right)$. Control experiments in DMSO and in other media with and without the presence of Tween 20 did not alter the $\mathrm{D}_{\text {confocal }}$ values significantly, consistent with the observed solubility of quinacrine across multiple solutions. Measuring the binding affinity of quinacrine to BSA, HEWL and myoglobin showed affinities of $4.6 \pm 1.1 \mu \mathrm{M}$, 4.7 $\pm 0.8 \mu \mathrm{M}$ and 3.0 $\pm 0.4 \mu \mathrm{M}$ (Figure $7 \mathrm{E}-\mathrm{G}$, Table S1).

As in the experiments, computed diffusion coefficients for quinacrine show no significant difference on adding $50 \mathrm{mg} / \mathrm{mL}$ protein crowder (Figure S1). One reason for the insensitivity to protein crowders is that quinacrine-protein contacts are less prominent compared to the other small molecules in BD simulations (Figure S3), as also shown by the absence of a peak in the quinacrine-HEWL RDF (Figure S4). However, the number of quinacrine-protein contacts was similar to that for the other small molecules for myoglobin and contacts were more pronounced for BSA. These differences are consistent with the larger positive charge on quinacrine than the other molecules $(+2 \mathrm{e})$, resulting in greater solubility in aqueous solution and a net charge repulsion to HEWL and a net charge attraction to BSA, which however does not result in any significant difference in either the computed or the experimentally determined diffusion coefficients for quinacrine in 50 $\mathrm{mg} / \mathrm{mL}$ BSA.

\section{Discussion:}

We used high-content screening of over 1000 drugs and the database fluorophores.org to search for drugs that are fluorescent in visible light, allowing us to follow their diffusion using FRAP. Of those identified, we chose fluorescein, doxorubicin, SB216763, and quinacrine for this study, as they have moderate to good quantum yields. Diffusion studies involving low MW drugs in multiple environments are rare, mostly as only a few drugs have the desired spectral properties. Still, studying their behavior in aqueous solutions is important as this is the medium for their administration and action. Here, we measured the diffusion of the four molecules in PBS and solutions containing one of three different 
proteins as crowders, BSA, HEWL, and myoglobin. In addition, we determined the affinity of the small molecule drugs and the three proteins using fluorescence quenching. The equilibrium dissociation constants are moderate (in the range of 1-100 $\mu \mathrm{M}$ ). These moderate affinities explain the lack of co-elution in size exclusion chromatography (SEC) of the small molecules to the protein crowders. Co-elution can be observed only for binding affinities better than $0.1 \mu \mathrm{M}$, which is not the case here (Figure S10, S11 and S12). Yet, the addition of the proteins had a substantial effect on the diffusion of the small molecules. BD simulations and molecular docking revealed mechanistic details of the main experimental observations, providing further evidence for the different mechanisms of modulation of small molecule diffusion rates observed.

Each of the four small molecules studied behaved differently, providing excellent examples for different types of diffusional behavior of small molecules (Figure 8). Quinacrine dihydrochloride is a cationic charged, water soluble compound. Its measured diffusion rate is not affected by the glass surface or the protein crowders. Fluorescein is a negatively charged, water-soluble compound. It forms interactions with proteins, which slow down its diffusion, apparently by forming protein-fluorescein oligomeric structures (Figure 4G). The opposite is observed for DOX, a positively charged molecule, which diffuses slowly in PBS, but at a faster rate on the addition of protein-crowders. A detailed analysis showed that DOX adsorbs to the glass surface and this is the reason for the slow diffusion. Last, SB216763 is a neutral molecule that is insoluble in water and forms large MW aggregates. However, addition of BSA as carrier protein solubilizes SB216763, as observed from micrographs, the fraction of recovery, the increased diffusion rate, and its ability to dock in a rather buried binding site on BSA.

A point of potential concern is the "slow" measured diffusion coefficients for the small molecules, which are much below those expected. A possible reason could be limitations in the line-FRAP method, which may not allow the measurement of high diffusion coefficients $\left(>100 \mu \mathrm{m}^{2} \mathrm{~s}^{-1}\right)$. If this would be a result of a "dead-time" between the end of the bleach and the first recovery data points, we should observe an incomplete bleach (as some recovery occurs before the first data measurement). However, the FRAP curves in Figures 2, 4, 6 and 7 do not show reduced bleach, even for the fastest diffusing molecules. This would suggest that for fast diffusing particles, the data analysis carried out 
on the experimental observations does not provide absolute diffusion coefficients.

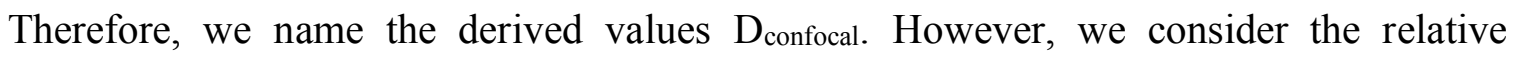
diffusion rates between the different conditions applied in this study to be robust, as the same data analysis procedure was carried out for all conditions. Unfortunately, methods such as FCS, which could provide an orthogonal approach for diffusion measurements, are not applicable to the small molecules used here, due to their low quantum yield.

In summary, by combining experiments and simulations, we see that the effects of protein crowders on the diffusion of small molecules go beyond the slowing of translational diffusion due to excluded volume, and find that the diffusion of drugs can also be slowed down due to quinary interactions, or it can be increased by surface detachment or a reduction in aggregation of the small molecules (Figure 8).

\section{Materials and Methods:}

BSA, myoglobin, and HEWL were purchased from Sigma-Aldrich. Doxorubicin was purchased from AdooQ Bioscience (catalog no. A14403). Fluorescein disodium salt was purchased from chemcruz (catalog no. sc-206026). The GSK3 inhibitor SB216763 (catalog no. ab120202), and quinacrine dihydrochloride (catalog no. ab120749), were purchased from Abcam. All other reagents used are described in a table format (Table S5, Key resources).

\section{Confocal microscopy and FRAP Analysis:}

Confocal microscopy. Images were collected with an Olympus IX81 FluoView FV1000 Spectral/SIM Scanner confocal laser-scanning microscope, using 60X DIC oil-immersion objective, N.A. 1.35. For fluorescein disodium salt fluorescence measurements, excitation was done at $440 \mathrm{~nm}$, using a diode laser at an output power of 1-4\% of maximal intensity for high to low concentrations, whereas emission was recorded from 520 to $550 \mathrm{~nm}$ using the spectral detection system. For doxorubicin, excitation was done at $515 \mathrm{~nm}$ diode laser using 5-10\% of the maximal intensity, and emission were collected from 540-640 nm. For quinacrine di hydro chloride and GSK3 inhibitor, excitation was done at $488 \mathrm{~nm}$ laser using $1-2 \%$ of the maximal intensity, while emission was collected from 502-560 nm with SDM560 emission dichromator cut off filter. All image analyses were performed using 
FluoView software, and data analyses were performed using Kaleidagraph software version 4.1 (Synergy).

\section{Line-FRAP and Classical XY-FRAP}

Line-FRAP was carried out in liquid drops. For photobleaching, "Tornado" of 4 pixels (2x2) diameter was used in the simultaneous stimulus scanner. This is the smallest area achievable using Tornado. The circle area of the bleach was kept precisely in the middle of the scanning line. The unidirectional lines were scanned with time intervals of $1.256 \mathrm{~ms}$ 1000 times (equivalent to $1.256 \mathrm{~s}$ ) in the majority of the measurements. The number of scans before, during, and after photobleaching was 10, 42, and 948, respectively. Photobleaching was achieved by the simultaneous laser at 405-nm excitations with 63 millisecond durations, using at full intensity (100\%). The simultaneous scanner moved at a speed of $100 \mu \mathrm{s} /$ pixel to perform an efficient photobleach. We have used two simultaneous scanners during the FRAP experiments: one scanner (at $405 \mathrm{~nm}$ with the full intensity of $100 \%$ ) for photobleaching and another scanner (at 440/515nm with weak intensity) for data acquisition. For all the drugs, bleach was performed by $405 \mathrm{~nm}$ laser, whereas for main excitations, Fluorescein $440 \mathrm{~nm}$ laser (1-4\%); GSK3 inhibitor $440 \mathrm{~nm}$ laser (0-1\%) were used. Emission collections were done from 520-550 nm for Quinacrine DHC and GSK3 inhibitor. Using the Olympus IX81 FluoView FV1000 Spectral/SIM Scanner confocal laser-scanning microscope, using Tornado (which requires SIM scanner to be loaded) greatly enhances bleaching efficiency. In addition, it shortens the time to obtain the first measurement after bleach (which is immediate in this mode). This property is highly beneficial for Line-FRAP measurements, where the time scale of data acquisition plays an important role. The Fluoview SIM scanner unit synchronizes laser light simulation with confocal and multiphoton imaging to avoid interruption to image observation during laser stimulation or manipulation. We have varied the intensity of the lasers to achieve a good signal/noise ratio. Fluorescence recovery plots were fitted to a double exponent growth curve. FRAP experiments were also performed inside the PBS buffer drops and in crowding conditions. Glass plate dish containing cover slips are used for microscopic measurements. Our previously developed Line FRAP protocol was employed for the final calculations of diffusion coefficients from the FRAP rates and averaged bleach sizes(45). 


\section{Steady-State Fluorescence Quenching Assays:}

Steady-state fluorescence quenching experiments were carried out on a Tecan fluorescence plate reader instrument. BSA, Myoglobin, and HEWL solutions of $2 \mu \mathrm{M}$ strength in PBS $1 \mathrm{X}(\mathrm{pH}=7.4)$ were prepared. Different small molecules are added in the protein solutions, maintaining the final concentrations between 1 to $100 \mu \mathrm{M}$. The final volume of each mixture was strictly $200 \mu \mathrm{L}$. The whole set of experiments was performed in a 96-well microplate system (black, flat bottom, Fluotrac). The fluorescence emission spectra were recorded at $\lambda_{\text {exc }}=280 \mathrm{~nm}$ and $\lambda_{\mathrm{em}}$ from 300 to $450 \mathrm{~nm}$. According to previous literature reports, the intensity at $344 \mathrm{~nm}$ (tryptophan) was used to calculate the dissociation constant or association constant $(59,60)$. The most generally valid equation to analyze fluorescence changes upon formation of a 1:1 complex is given by Eq. (2)

$$
\frac{F_{0}-F}{F_{0}-F_{c}}=\frac{[P Q]}{[P]_{t}}=\frac{[P]_{t}+[Q]_{a}+K_{d}-\sqrt{\left([P]_{t}+[Q]_{a}+K_{d}\right)^{2}-4[P]_{t}[Q]_{a}}}{2[P]_{t}}
$$

Where $F$ is the measured fluorescence, $F_{0}$ is the starting fluorescence, $F_{c}$ is the fluorescence of the fully complexed protein, $K_{\mathrm{d}}$ the dissociation constant, $[P]_{\mathrm{t}}$ the concentration of protein, and $[Q]_{\mathrm{a}}$ is the concentration of added ligand/quencher (small molecule/drug in our case). In Eq. (2), it should be noted that $K_{\mathrm{d}}\left(1 / K_{\mathrm{a}}\right)$ is used instead of $K_{\mathrm{a}}$. This is an equation with two unknowns, $K_{\mathrm{d}}$ and $F_{\mathrm{c}}$, which most mathematical software programmes can solve. We used Kaleidagraph software version 4.1 (Synergy) to solve the equation. The dissociation constants, $K_{\mathrm{d}}$, are given in Table $\mathrm{S} 1$.

\section{Brownian dynamics simulations}

The initial coordinates for HEWL, BSA and myoglobin were obtained from the PDB files 1HEL (61), 4F5S (62) and 1DWR (63), respectively. Partial atomic charges and protonation states for the protein crowders at $\mathrm{pH} 7.2$ were computed using pdb2pqr (64, 65). Partial atomic charges for the heme group of myoglobin were obtained from previous work (66).

The initial coordinates for the small molecules were obtained from conversion of SMILES to PDB format using Babel (67). The protonation state of the molecules at $\mathrm{pH} 7.2$ was determined using Epik (68) in Maestro (Schrödinger, LLC, New York, NY). The 
structures of the four small molecules were energy minimized using Maestro and then submitted to quantum mechanical calculations in GAMESS (69) using HF and the 6-31G** basis set to obtain RESP $(70,71)$ partial atomic charges.

BD simulations were performed using the Simulation of Diffusional Association (SDA) 7 software package (72), version 7.2 available at https://mcm.h-its.org/sda. All simulation boxes had the same composition: 80 small molecules of one type (fluorescein, doxorubicin, quinacrine or GSK3 inhibitor), or 80 small molecules and 440 protein crowders of one type (BSA, HEWL or myoglobin), resulting in a crowder:small molecule ratio of 5.5, which mimics the experimental conditions. Different crowder concentrations were achieved by changing the size of the cubic periodic simulation box (Table S6).

Three replica BD simulations were performed for each system. BD simulations were performed using a time step of $0.5 \mathrm{ps}$, ionic strength of $190 \mathrm{mM}$ and periodic boundary conditions. Small molecules and proteins were kept rigid, and the same conformations of each small molecule and protein were used for all simulations. The translational and rotational diffusion coefficients of the small molecules and proteins at infinite dilution in aqueous solution, necessary to perform BD simulations, were computed from HYDROPRO (73), with a radius of the atomic element (AER) of $2.9 \AA$ for proteins and $1.2 \AA$ for small molecules (see details below, Table S7). Hydrodynamic interactions were computed using a mean-field model (38). The Stokes radii of the small molecules and proteins, necessary to compute hydrodynamic interactions, were calculated from the solvent-accessible volume estimated from a single point Poisson-Boltzmann calculation performed using AMBER 2016 (74) (Table S2). A radius of four times the Stokes radius of the protein was used to define the local volume for computing hydrodynamic interactions. The forces between molecules were modelled by computing electrostatic interaction, electrostatic desolvation and non-polar desolvation terms from the interactions between the atoms of each molecule and precomputed potential grids on the other molecules. The grid spacing for protein crowders and small molecules was $0.75 \AA$ for simulations with doxorubicin and $0.65 \AA$ for simulations with the other small molecules. The lengths of the sides of the cubic grids for the small molecules were 97 grid points (electrostatic potential) and 80 grid points (for the other, shorter-range potentials). The lengths of the sides of the cubic grids were 161 grid points (electrostatic potential) and grid 
135 points (for the other potentials) for HEWL, 225 grid points (electrostatic potential) and 193 grid points (for the other potentials) for BSA, and 225 grid points (electrostatic potential) and 200 grid points (for the other potentials) for myoglobin.

First, all simulated systems were subjected to a $0.2 \mu$ s-length BD simulation with interactions between molecules modelled by only a soft-core repulsion energy term in order to resolve any steric clashes in the initial simulation box generated by the SDA tool genbox. After this, BD production simulations were performed for $10 \mu \mathrm{s}$.

The parameters and grids described above were also employed to perform BD simulations in the presence of a silica surface to mimic glass. The parameters for the silica surface were obtained from a previous BD simulation study (40), and a similar simulation setup was used here. The silica surface was represented as a homogeneously charged graphite lattice surface with a charge density of $-0.0013 \mathrm{e} / \AA^{2}$. The surface was assigned a charge distribution of -0.0032 e/atom. The $\mathrm{x}$ and $\mathrm{y}$ dimensions of the simulation box and of the silica surface were $340 \AA$. The desired crowder concentration was achieved by changing the height ( $\mathrm{z}$ axis) of the simulation box. In the starting configuration for $\mathrm{BD}$ simulations, a buffer region of $120 \AA$ separated the surface and the solutes. Before inclusion of the surface and buffer region in the system, systems were subjected to an initial $0.2 \mu \mathrm{s}-$ length $\mathrm{BD}$ simulation with interactions between molecules modelled by only a soft-core repulsion energy term in order to resolve any steric clashes in the initial simulation box. After this, BD production simulations were performed for $10 \mu \mathrm{s}$.

In the analysis of molecule adsorption, a molecule was considered adsorbed when its distance from the surface was less than 4 times the Stokes radius of the molecule, as done previously (40).

For all BD simulations, radial distribution functions and the number of small molecule-protein or small molecule-small molecule contact interactions were computed using SDA7. Contacts were defined as present when heavy atoms (at least one in each molecule) were within a distance of $4.5 \AA$ of each other. Snapshots were analysed at intervals of 500 picoseconds and values averaged over the last 10 microseconds of the simulations.

\section{Computation of diffusion coefficients for small molecules}


HYDROPRO(73) was parameterized to reproduce the experimental translational and rotational diffusion coefficients of proteins. One parameter, the radius of the atomic elements (AER), was adjusted to reproduce the experimental results. A procedure similar to that followed in the original publication (73) was followed to obtain an AER value for small molecules:

1. estimate translational diffusion coefficients using varying values of AER $(1,2,3$ and 4 A);

2. do a linear fit for the graph AER values vs. translational diffusion coefficients for each small molecule, and calculate the AER value that reproduces the experimental translational diffusion coefficient;

3. calculate the final AER value as the average of the AER values obtained for each small molecule.

Following the procedure above, an AER of $1.2 \AA$ was obtained for small molecules. Table S7 shows the computed diffusion coefficients for molecules with known experimental diffusion coefficients using AER values before and after the reparameterization for small molecules.

\section{Docking of the small molecules to the proteins}

Docking was performed using Autodock Vina (75), a cubic grid with a spacing of $0.375 \AA$ and 70 grid points and default parameters. The grid was centered on the catalytic site of HEWL (PDB 1HEL) or on one of the four binding cavities of BSA (PDB 4F5S), identified by the presence of 3,5-diiodosalicylic acid in the complex with BSA in PDB 4JK4 (76). The docking pose with the highest score was retained.

\section{Acknowledgments}

We gratefully acknowledge Stefan Richter (HITS) for technical support of the computational work. This work was supported by the Israel Science Foundation grant no. 1268/18 (GS), a Capes-Humboldt postdoctoral scholarship to AN-A (Capes process number 88881.162167/2017-01), funds from the Cluster of Excellence CellNetworks (DFG, EXC81) to AN-A, the European Union's Horizon 2020 Framework Programme for 
Research and Innovation under Grant Agreements 785907 and 945539 (Human Brain Project SGA2 and SGA3) (RCW), and the Klaus Tschira Foundation (AN-A, RCW).

\section{REFERENCES:}

1. C. A. Lipinski, F. Lombardo, B. W. Dominy, P. J. Feeney, Experimental and computational approaches to estimate solubility and permeability in drug discovery and development settings. Adv. Drug Deliv. Rev. 23, 3-25 (1997).

2. S. Schreier, S. V. P. Malheiros, E. De Paula, Surface active drugs: self-association and interaction with membranes and surfactants. Physicochemical and biological aspects. Biochim. Biophys. Acta - Biomembr. 1508, 210-234 (2000).

3. D. Curry, et al., Prevention of doxorubicin sorptive losses in drug delivery studies using polyethylene glycol. RSC Adv. 5, 25693-25698 (2015).

4. B. Y. Feng, et al., A high-throughput screen for aggregation-based inhibition in a large compound library. J. Med. Chem. 50, 2385-2390 (2007).

5. B. Y. Feng, B. K. Shoichet, A detergent-based assay for the detection of promiscuous inhibitors. Nat. Protoc. 1, 550-553 (2006).

6. N. Thorne, D. S. Auld, J. Inglese, Apparent activity in high-throughput screening: origins of compound-dependent assay interference. Curr. Opin. Chem. Biol. 14, 315-324 (2010).

7. S. C. Owen, A. K. Doak, P. Wassam, M. S. Shoichet, B. K. Shoichet, Colloidal aggregation affects the efficacy of anticancer drugs in cell culture. ACS Chem. Biol. 7, 1429-1435 (2012).

8. S. B. Zimmerman, S. O. Trach, Estimation of macromolecule concentrations and excluded volume effects for the cytoplasm of Escherichia coli. J. Mol. Biol. 222, 599-620 (1991).

9. R. P. Ribeiro, J. T. S. Coimbra, M. J. Ramos, P. A. Fernandes, Diffusion of the small, very polar, drug piracetam through a lipid bilayer: an MD simulation study. Theor. Chem. Acc. 136, 46 (2017).

10. L. Di, et al., Evidence-based approach to assess passive diffusion and carriermediated drug transport. Drug Discov. Today 17, 905-912 (2012).

11. P. D. Dobson, D. B. Kell, Carrier-mediated cellular uptake of pharmaceutical drugs: An exception or the rule? Nat. Rev. Drug Discov. 7, 205-220 (2008).

12. K. Sugano, et al., Coexistence of passive and carrier-mediated processes in drug transport. Nat. Rev. Drug Discov. 9, 597-614 (2010).

13. S. Miyamoto, K. Shimono, Molecular Modeling to Estimate the Diffusion Coefficients of Drugs and Other Small Molecules. Molecules 25, 5340 (2020).

14. P. M. Kekenes-Huskey, C. Eun, J. A. McCammon, Enzyme localization, crowding, and buffers collectively modulate diffusion-influenced signal transduction: Insights from continuum diffusion modeling. J. Chem. Phys. 143, 094103 (2015). 
15. J. Miguel Rubi, Entropic diffusion in confined soft-matter and biological systems. Epl 127, 10001 (2019).

16. M. Feig, G. Nawrocki, I. Yu, P. H. Wang, Y. Sugita, Challenges and opportunities in connecting simulations with experiments via molecular dynamics of cellular environments in Journal of Physics: Conference Series, 1036, 012010 (2018).

17. N. S. White, R. J. Errington, Fluorescence techniques for drug delivery research: Theory and practice. Adv. Drug Deliv. Rev. 57, 17-42 (2005).

18. Y. Zhang, K. Y. Park, K. F. Suazo, M. D. Distefano, Recent progress in enzymatic protein labelling techniques and their applications. Chem. Soc. Rev. 47, 9106-9136 (2018).

19. J. Robert, L. Gianni, Pharmacokinetics and metabolism of anthracyclines. Cancer Surveys, 17, 153-165 (1993).

20. C. Carvalho, et al., Doxorubicin: The Good, the Bad and the Ugly Effect. Curr. Med. Chem. 16, 3267-3285 (2009).

21. R. Ehsanian, C. Van Waes, S. M. Feller, Beyond DNA binding - A review of the potential mechanisms mediating quinacrine's therapeutic activities in parasitic infections, inflammation, and cancers. Cell Commun. Signal. 9, 13 (2011).

22. K. Gurova, New hopes from old drugs: Revisiting DNA-binding small molecules as anticancer agents. Futur. Oncol. 5, 1685-1704 (2009).

23. M. P. Coghlan, et al., Selective small molecule inhibitors of glycogen synthase kinase-3 modulate glycogen metabolism and gene transcription. Chem. Biol. 7, 793-803 (2000).

24. A. Wagman, K. Johnson, D. Bussiere, Discovery and Development of GSK3 Inhibitors for the Treatment of Type 2 Diabetes. Curr. Pharm. Des. 10, 11051137 (2005).

25. M. M. Brooks, S. Neelam, P. R. Cammarata, Lenticular mitoprotection. Part B: GSK-3 $\beta$ and regulation of mitochondrial permeability transition for lens epithelial cells in atmospheric oxygen. Mol. Vis. 19, 2451-2467 (2013).

26. B. Zhitomirsky, Y. G. Assaraf, Lysosomal sequestration of hydrophobic weak base chemotherapeutics triggers lysosomal biogenesis and lysosome dependent cancer multidrug resistance. Oncotarget 6, 1143-1156 (2015).

27. B. K. Lipson, L. A. Yannuzzi, Complications of intravenous fluorescein injections. Int. Ophthalmol. Clin. 29, 200-205 (1989).

28. K. I. O'goshi, J. Serup, Safety of sodium fluorescein for in vivo study of skin. Ski. Res. Technol. 12, 155-161 (2006).

29. A. C. Miklos, M. Sarkar, Y. Wang, G. J. Pielak, Protein crowding tunes protein stability. J. Am. Chem. Soc. 133, 7116-7120 (2011).

30. M. Feig, Y. Sugita, Variable interactions between protein crowders and biomolecular solutes are important in understanding cellular crowding. J. Phys. Chem. B 116, 599-605 (2012).

31. J. Seidler, S. L. McGovern, T. N. Doman, B. K. Shoichet, Identification and prediction of promiscuous aggregating inhibitors among known drugs. J. Med. Chem. 46, 4477-4486 (2003). 
32. A. K. Doak, H. Wille, S. B. Prusiner, B. K. Shoichet, Colloid formation by drugs in simulated intestinal fluid. J. Med. Chem. 53, 4259-4265 (2010).

33. G. A. Huber, J. A. McCammon, Brownian Dynamics Simulations of Biological Molecules. Trends Chem. 1, 727-738 (2019).

34. J. T. Bullerjahn, S. Von Bülow, G. Hummer, Optimal estimates of self-diffusion coefficients from molecular dynamics simulations. J. Chem. Phys. 153, 024116 (2020).

35. S. Von Bülow, J. T. Bullerjahn, G. Hummer, Systematic errors in diffusion coefficients from long-time molecular dynamics simulations at constant pressure. J. Chem. Phys. 153, 021101 (2020).

36. K. Kasahara, et al., Reduced efficacy of a Src kinase inhibitor in crowded protein solution. Nat. Commun. 12, 4099 (2021).

37. J. Balbo, P. Mereghetti, D. P. Herten, R. C. Wade, The shape of protein crowders is a major determinant of protein diffusion. Biophys. J. 104, 1576-1584 (2013).

38. P. Mereghetti, R. C. Wade, Atomic detail brownian dynamics simulations of concentrated protein solutions with a mean field treatment of hydrodynamic interactions. J. Phys. Chem. B 116, 8523-8533 (2012).

39. J. Romanowska, D. B. Kokh, R. C. Wade, When the Label Matters: Adsorption of Labeled and Unlabeled Proteins on Charged Surfaces. Nano Lett. 15, 7508-7513 (2015).

40. M. Reinhardt, N. J. Bruce, D. B. Kokh, R. C. Wade, Brownian Dynamics Simulations of Proteins in the Presence of Surfaces: Long-Range Electrostatics and Mean-Field Hydrodynamics. J. Chem. Theory Comput. 17, 3510-3524 (2021).

41. N. Lorén, et al., Fluorescence recovery after photobleaching in material and life sciences: Putting theory into practice. Q. Rev. Biophys. 48, 323-387 (2015).

42. E. Dauty, A. S. Verkman, Molecular crowding reduces to a similar extent the diffusion of small solutes and macromolecules: Measurement by fluorescence correlation spectroscopy. Journal of Molecular Recognition, 17, 441-447 (2004).

43. S. Manley, et al., High-density mapping of single-molecule trajectories with photoactivated localization microscopy. Nat. Methods 5, 155-157 (2008).

44. H. Deschout, K. Raemdonck, J. Demeester, S. C. De Smedt, K. Braeckmans, FRAP in pharmaceutical research: Practical guidelines and applications in drug delivery. Pharm. Res. 31, 255-270 (2014).

45. D. Dey, et al., Line-FRAP, A Versatile Method to Measure Diffusion Rates In Vitro and In Vivo. J. Mol. Biol. 433, 166898 (2021).

46. M. Hoffmann, C. S. Wagner, L. Harnau, A. Wittemann, 3D brownian diffusion of submicron-sized particle clusters. ACS Nano 3, 3326-3334 (2009).

47. D. Axelrod, D. E. Koppel, J. Schlessinger, E. Elson, W. W. Webb, Mobility measurement by analysis of fluorescence photobleaching recovery kinetics. Biophys. J. 16, 1055-1069 (1976).

48. D. M. Soumpasis, Theoretical analysis of fluorescence photobleaching recovery experiments. Biophys. J. 41, 95-97 (1983). 
49. S. von Bülow, M. Siggel, M. Linke, G. Hummer, Dynamic cluster formation determines viscosity and diffusion in dense protein solutions. Proc. Natl. Acad. Sci. U. S. A. 116, 9843-9852 (2019).

50. D. Agudelo, et al., Probing the binding sites of antibiotic drugs doxorubicin and $\mathrm{N}$-(trifluoroacetyl) doxorubicin with human and bovine serum albumins. PLoS One 7 e43814 (2012).

51. Z. Fülöp, R. Gref, T. Loftsson, A permeation method for detection of selfaggregation of doxorubicin in aqueous environment. Int. J. Pharm. 454, 559-561 (2013).

52. K. Kubiak-Ossowska, K. Tokarczyk, B. Jachimska, P. A. Mulheran, Bovine Serum Albumin Adsorption at a Silica Surface Explored by Simulation and Experiment. J. Phys. Chem. B 121, 3975-3986 (2017).

53. A. Diéz Fernández, P. Charchar, A. G. Cherstvy, R. Metzler, M. W. Finnis, The diffusion of doxorubicin drug molecules in silica nanoslits is non-Gaussian, intermittent and anticorrelated. Phys. Chem. Chem. Phys. 22, 27955-27965 (2020).

54. K. Kubiak-Ossowska, M. Cwieka, A. Kaczynska, B. Jachimska, P. A. Mulheran, Lysozyme adsorption at a silica surface using simulation and experiment: Effects of $\mathrm{pH}$ on protein layer structure. Phys. Chem. Chem. Phys. 17, 24070-24077 (2015).

55. C. N. Nassab, et al., Lysozyme and Human Serum Albumin Proteins as Potential Nitric Oxide Cardiovascular Drug Carriers: Theoretical and Experimental Investigation. J. Phys. Chem. B 125, 7750-7762 (2021).

56. E. Fliszár-Nyúl, et al., Interaction of mycotoxin alternariol with serum albumin. Int. J. Mol. Sci. 20, 2352 (2019).

57. M. S. Rojas, et al., Quinacrine, an antimalarial drug with strong activity inhibiting sars-cov-2 viral replication in vitro. Viruses 13, 121 (2021).

58. B. Pineda, V. P. D. La Cruz, H. R. Pando, J. Sotelo, Quinacrine as a potential treatment for COVID-19 virus infection. Eur. Rev. Med. Pharmacol. Sci. 25, 556566 (2021).

59. M. Van De Weert, L. Stella, Fluorescence quenching and ligand binding: A critical discussion of a popular methodology. J. Mol. Struct. 998, 144-150 (2011).

60. K. A. Bakar, S. R. Feroz, A critical view on the analysis of fluorescence quenching data for determining ligand-protein binding affinity. Spectrochim. Acta - Part A Mol. Biomol. Spectrosc. 223, 117337 (2019).

61. K. P. Wilson, B. A. Malcolm, B. W. Matthews, Structural and thermodynamic analysis of compensating mutations within the core of chicken egg white lysozyme. J. Biol. Chem. 267, 10842-10849 (1992).

62. A. Bujacz, Structures of bovine, equine and leporine serum albumin. Acta Crystallogr. Sect. D Biol. Crystallogr. 68, 1278-1289 (2012).

63. K. Chu, et al., Structure of a ligand-binding intermediate in wild-type carbonmonoxy myoglobin. Nature 403, 921-923 (2000). 
64. T. J. Dolinsky, J. E. Nielsen, J. A. McCammon, N. A. Baker, PDB2PQR: An automated pipeline for the setup of Poisson-Boltzmann electrostatics calculations. Nucleic Acids Res. 32, W665-W667 (2004).

65. S. Unni, et al., Web servers and services for electrostatics calculations with APBS and PDB2PQR. J. Comput. Chem. 32, 1488-1491 (2011).

66. D. A. Giammona, “An Examination of Conformational Flexibility in Porphyrins and Bulky-Ligand Binding in Myoglobin." (1984).

67. N. M. O’Boyle, et al., Open Babel: An Open chemical toolbox. J. Cheminform. 3, 1,33 (2011).

68. J. C. Shelley, et al., Epik: A software program for $\mathrm{pKa}$ prediction and protonation state generation for drug-like molecules. J. Comput. Aided. Mol. Des. 21, 681691 (2007).

69. C. E. Dykstra, G. Frenking, K. S. Kim, G. E. Scuseria, Theory and Applications of Computational Chemistry (2005).

70. C. I. Bayly, P. Cieplak, W. D. Cornell, P. A. Kollman, A well-behaved electrostatic potential based method using charge restraints for deriving atomic charges: The RESP model. J. Phys. Chem. 97, 10269-10280 (1993).

71. W. D. Cornell, P. Cieplak, C. I. Bayly, P. A. Kollman, Application of RESP Charges To Calculate Conformational Energies, Hydrogen Bond Energies, and Free Energies of Solvation. J. Am. Chem. Soc. 115, 9620-9631 (1993).

72. M. Martinez, et al., SDA 7: A modular and parallel implementation of the simulation of diffusional association software. J. Comput. Chem. 36, 1631-1645 (2015).

73. J. García De La Torre, M. L. Huertas, B. Carrasco, Calculation of hydrodynamic properties of globular proteins from their atomic-level structure. Biophys. J. 78, 719-730 (2000).

74. D. A. Case, et al., Amber16. Univ. California, San Fr. (2016)

75. O. Trott, A. J. Olson, AutoDock Vina: Improving the speed and accuracy of docking with a new scoring function, efficient optimization, and multithreading. J. Comput. Chem. 31, 455-461 (2009).

76. B. Sekula, K. Zielinski, A. Bujacz, Crystallographic studies of the complexes of bovine and equine serum albumin with 3,5-diiodosalicylic acid. Int. J. Biol.

Macromol. 60, 316-324 (2013). 
A $\mathrm{Na}$<smiles>[NH3+][NH3+]</smiles>

Fluorescein disodium Salt

B<smiles>COc1cccc2c1C(=O)c1c(O)c3c(c(O)c1C2=O)CC(O)(C(=O)CO)CC3OC1CC([NH3+])C(O)C(C)O1</smiles>

C

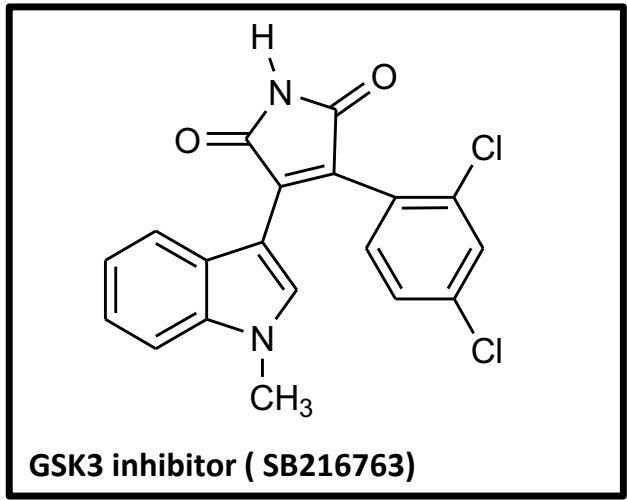

D<smiles>CCN(CC)CCCC(C)Nc1c2cc(OC)ccc2[n+]([O-])c2cc(Cl)ccc12</smiles>
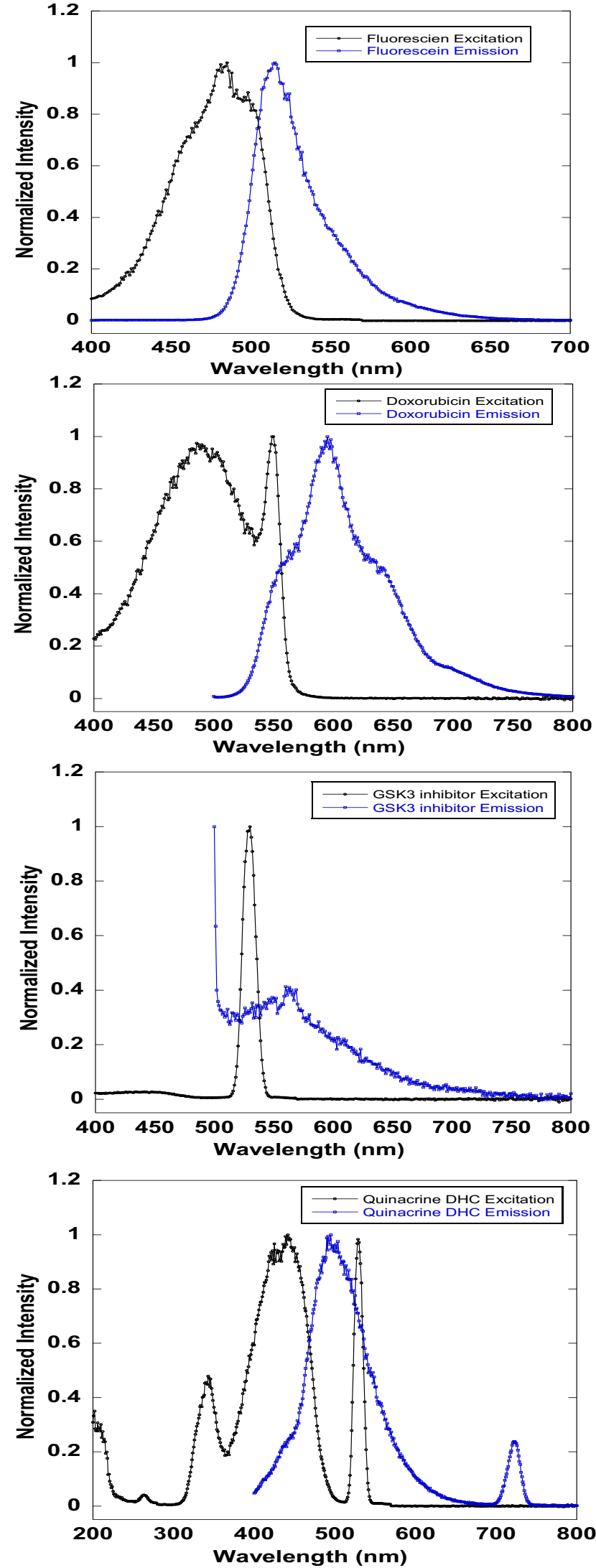

Figure 1: Chemical structures and comparative excitation and emission spectra with relative strengths:

(A) fluorescein disodium salt, (B) doxorubicin, (C) GSK3 SB216763 inhibitor, and (D) quinacrine dihydrochloride 
A

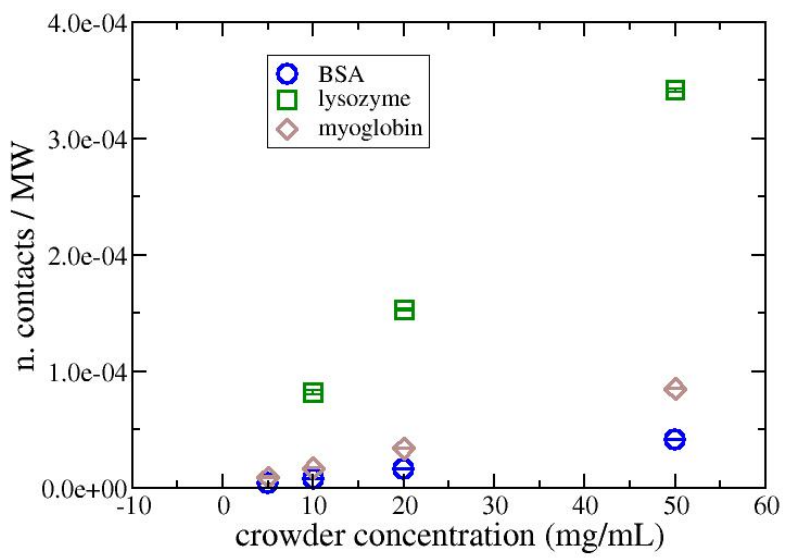

B

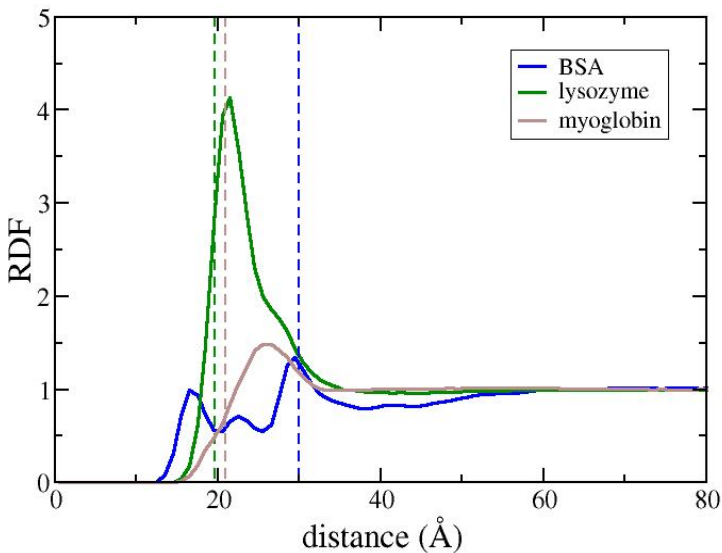

Figure 3: BD simulations reveal protein-drug interactions: Interactions between fluorescein and protein crowders in BD simulations show differences between the three protein crowders. The number of protein-fluorescein contact interactions and the RDF peak are higher in the presence of HEWL, indicating stronger interactions of fluorescein with this protein crowder. (A) Number of protein-fluorescein contact interactions divided by the molecular weight (MW) of the protein crowder (66637, 17820 and $14331 \mathrm{~g} / \mathrm{mol}$ for BSA, myoglobin and HEWL, respectively) plotted against protein crowder concentration. (B) Radial distribution functions (RDF) for proteinfluorescein distances computed from simulations with $50 \mathrm{mg} / \mathrm{mL}$ of crowder $(\mathrm{pH} 7.2$, ionic strength of $190 \mathrm{mM}$ ). The dashed lines indicate the sum of the Stokes radii of the protein crowder and of fluorescein. Stokes radii of proteins: $25.7 \AA, 16.6 \AA$ and $15.3 \AA$ for BSA, myoglobin and HEWL, respectively. Stokes radius of fluorescein: $4.3 \AA$. 
DOX Diffusion: Additive effects

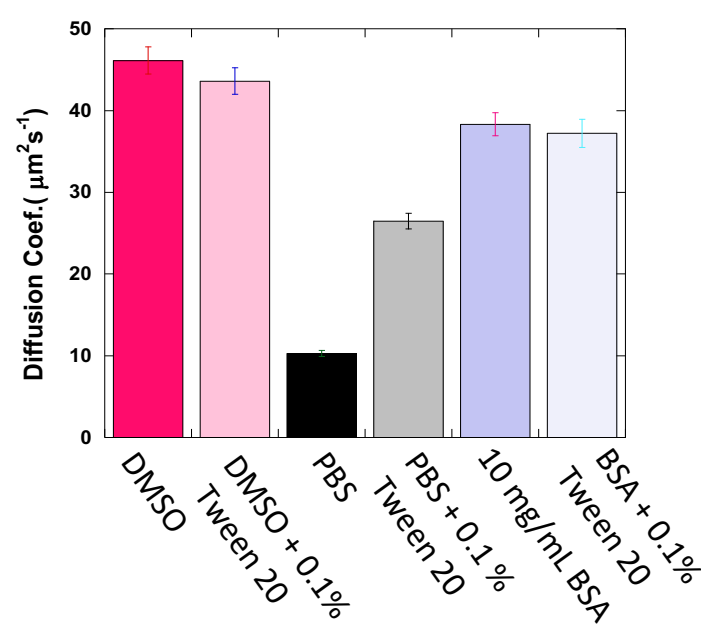

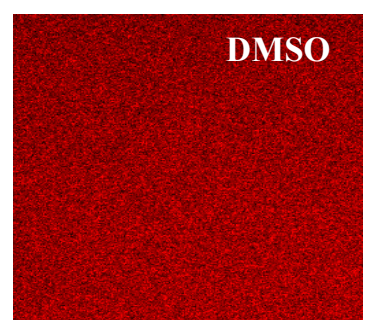

DMSO + Tween 20

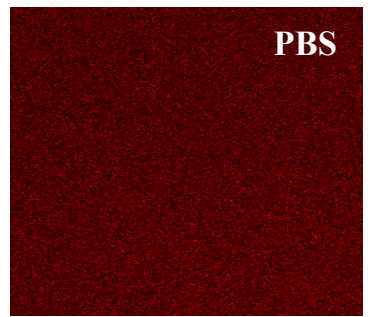

PBS + Tween 20

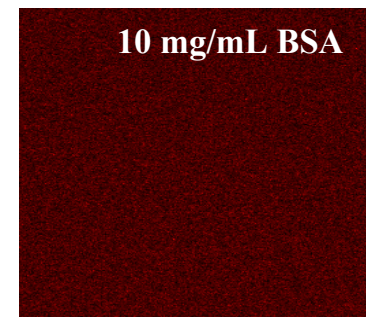

$10 \mathrm{mg} / \mathrm{mL} \mathrm{BSA}$

+ Tween 20

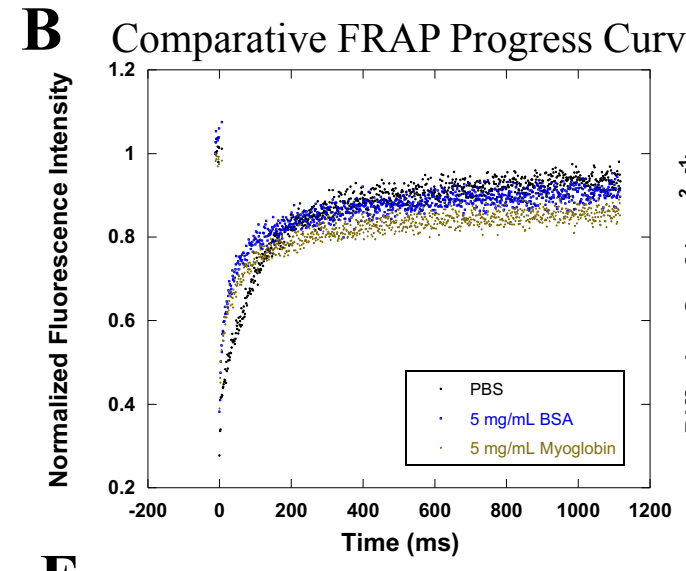

$\mathbf{E}$

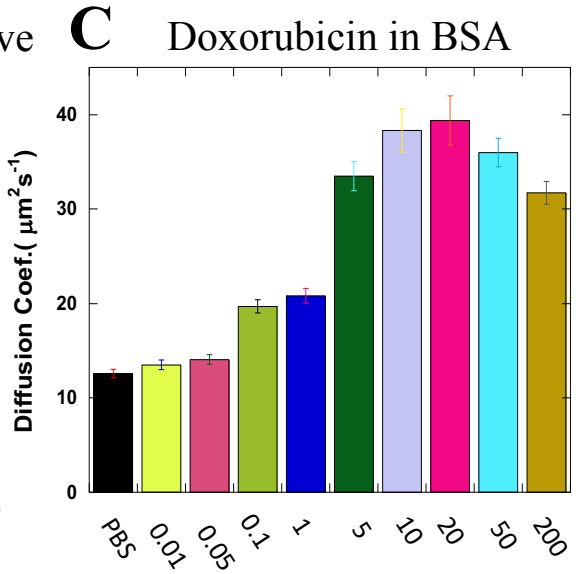

Conc. $(\mathrm{mg} / \mathrm{mL})$

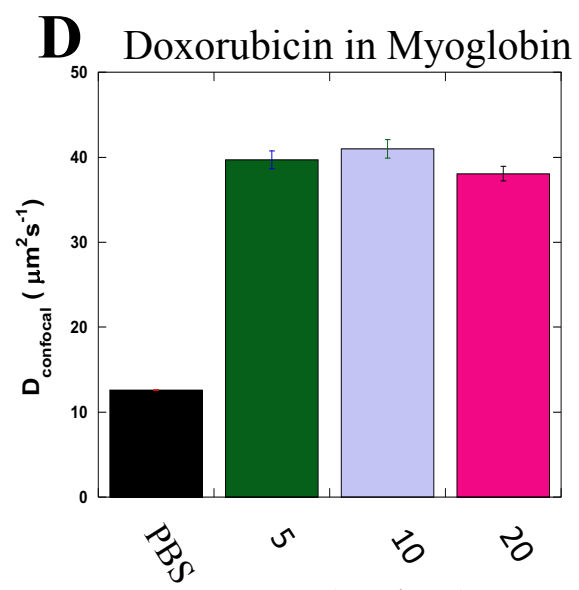

Conc. $(\mathrm{mg} / \mathrm{mL})$

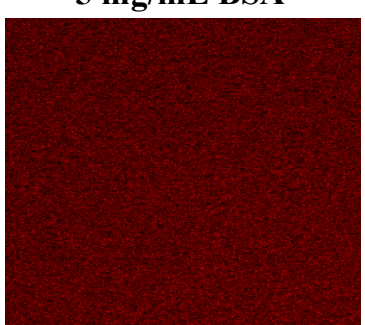

$5 \mathrm{mg} / \mathrm{mL}$ Myoglobin

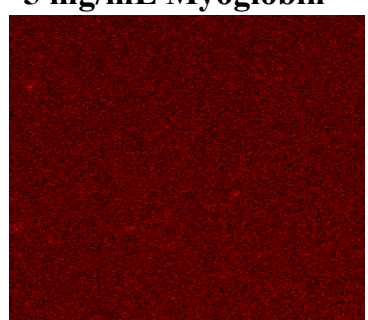

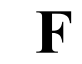

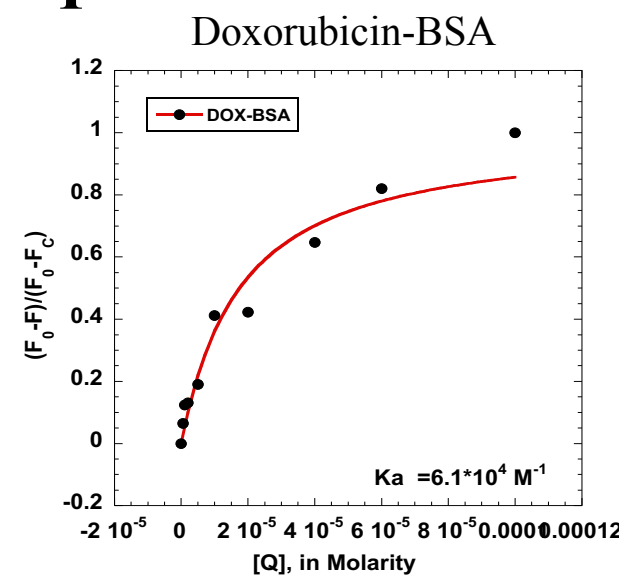

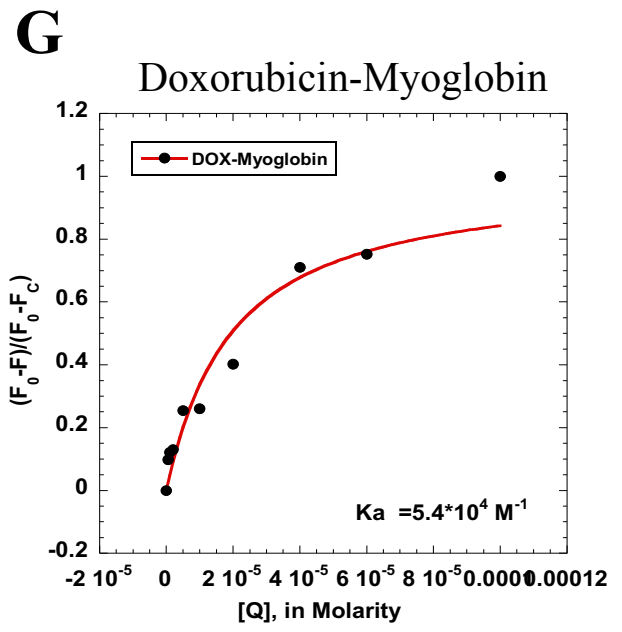

Figure 4: Doxorubicin in protein crowders: (A) Comparative diffusion coefficients and images from confocal microscope of doxorubicin in diverse environments with or without tween 20 are shown. (B) Comparative averaged FRAP profiles ( $N=30 ; R=0.99$ for each of the fits) and (E) images from confocal microscopes of doxorubicin in presence of $5 \mathrm{mg} / \mathrm{mL}$ of BSA and myoglobin are shown. Comparative diffusion coefficients of DOX in different amounts of (C) BSA and (D) Myoglobin are shown. $\left(\mathrm{F}_{0}-\mathrm{F}\right) /\left(\mathrm{F}_{0}-\mathrm{F}_{\mathrm{c}}\right)$ vs [Q] plots from fluorescence quenching experiments of $(F)$ DOX-BSA $(R=0.98),(G)$ DOX-Myoglobin $(R=0.97)$, systems in PBS buffer are shown, where $\mathrm{Q}$ is the titrating drug concentration in molarity. 
A

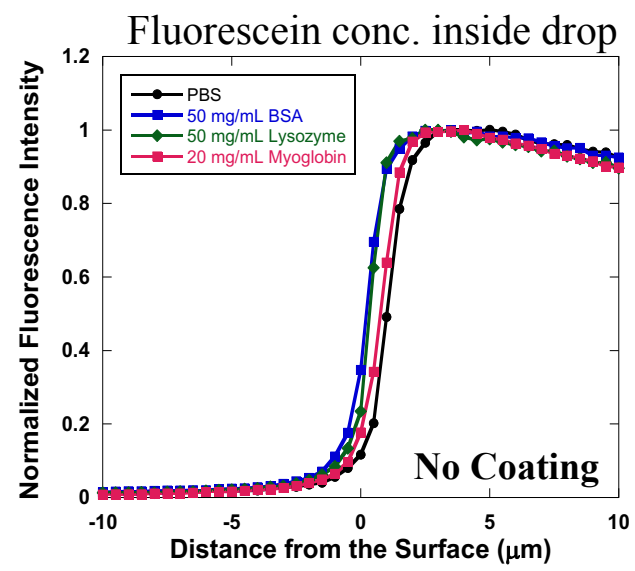

\section{Effect of Surface coating}

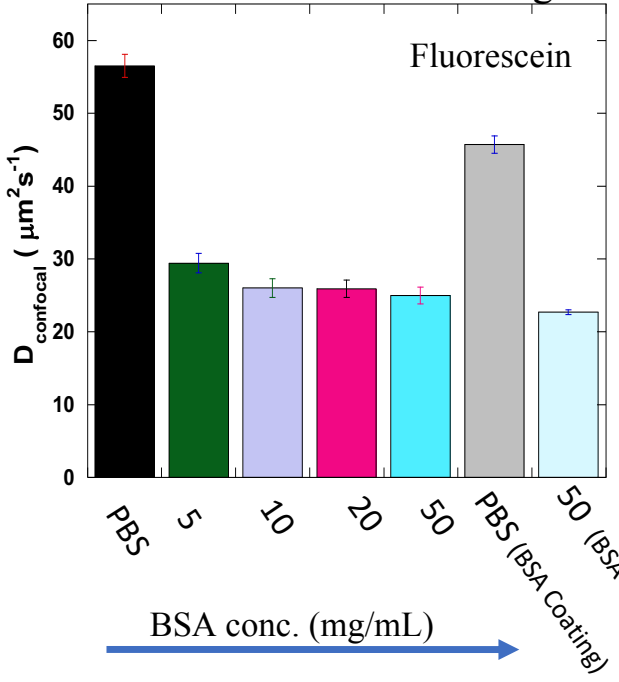

G

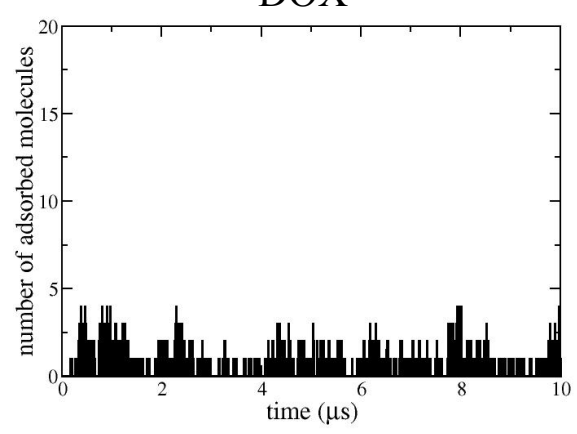

B
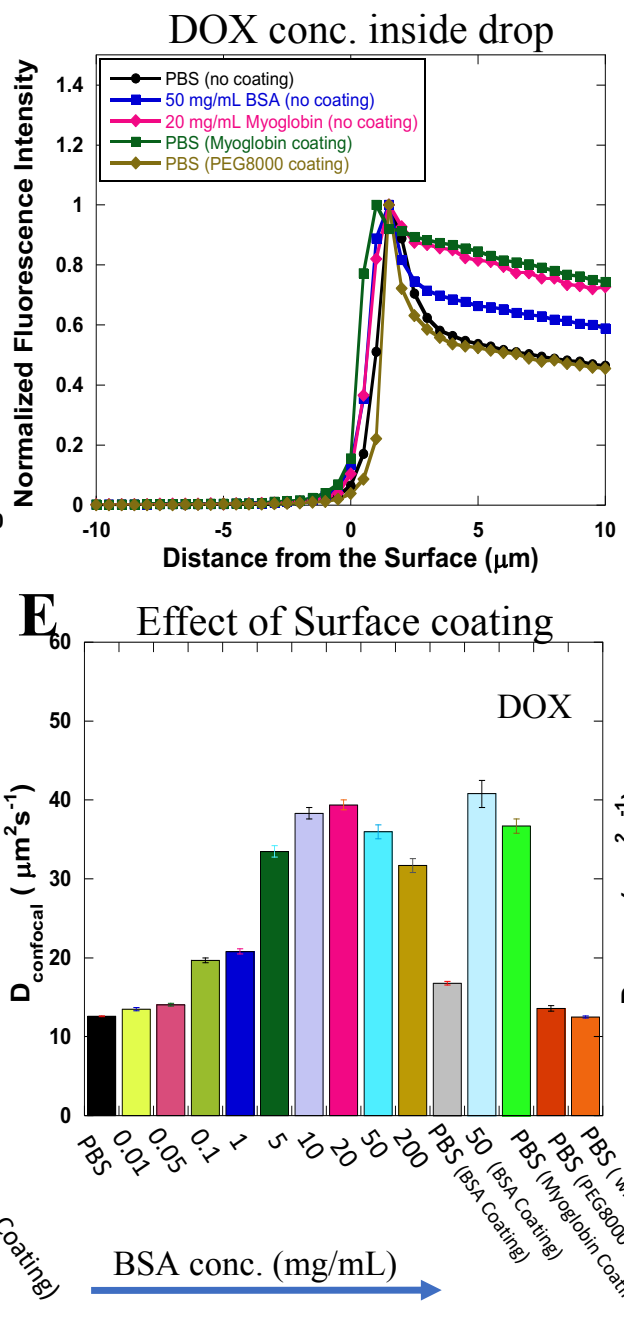

DOX

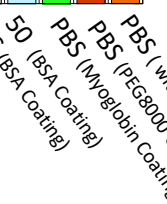

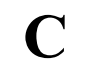

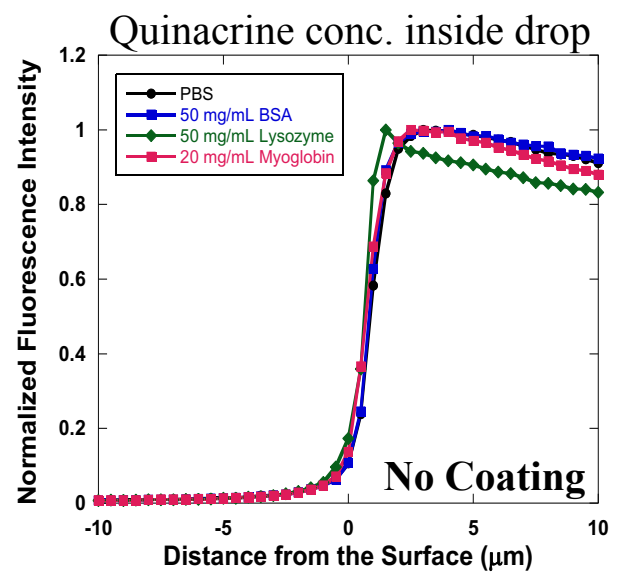

$\mathbf{F}$

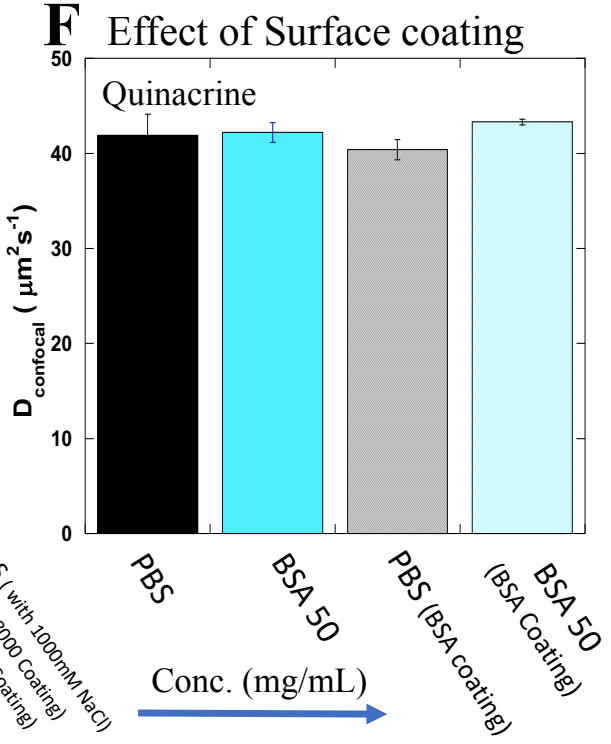

Figure 5: Adsorption of drugs and protein crowders to a glass surface. Determination of drop homogeneity of (A) Fluorescein (B) Doxorubicin and (C) Quinacrine in different crowding environments and in buffer solutions with or without surface coating. Effect of surface coating (by BSA or myoglobin) of glass plates on measured diffusion rates is shown for (D) Fluorescein, (E) Doxorubicin and (F) Quinacrine. (G) Number of doxorubicin or protein crowder molecules adsorbed to a silica surface in BD simulations performed with one type of solute molecule ( 80 molecules of DOX or 440 molecules of protein crowder at 50

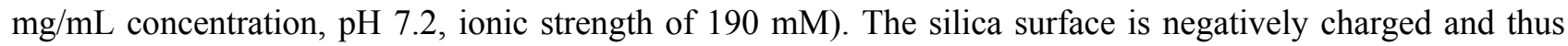
electrostatic interactions favor the binding of HEWL (+8 e) (see Figure S9) relative to BSA ( -16 e). Myoglobin ( -2 e) and DOX $(+1$ e) have small net charges and their electrostatic distribution (Figure S5) and ability to make hydrophobic interactions with the surface are important for their adsorption to the surface. 
(which was not certified by peer review) is the author/funder, who has granted bioRxiv a license to display the preprint in perpetuity. It is $m$

A PBS (1X ZOOM)

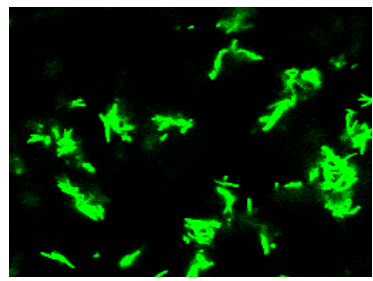

$25 \mathrm{mg} / \mathrm{mL}$ of BSA

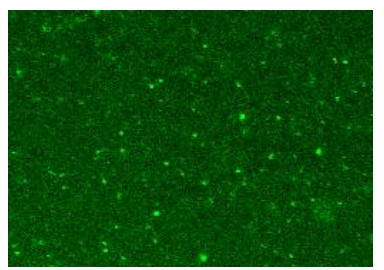

B

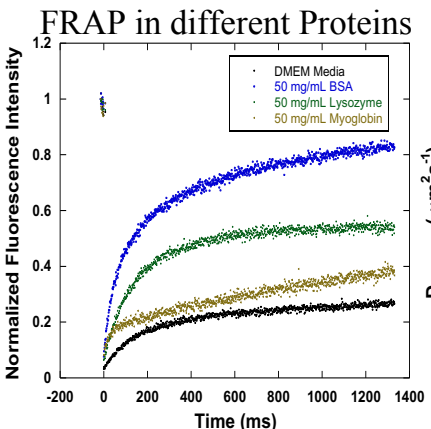

$\mathbf{F}$

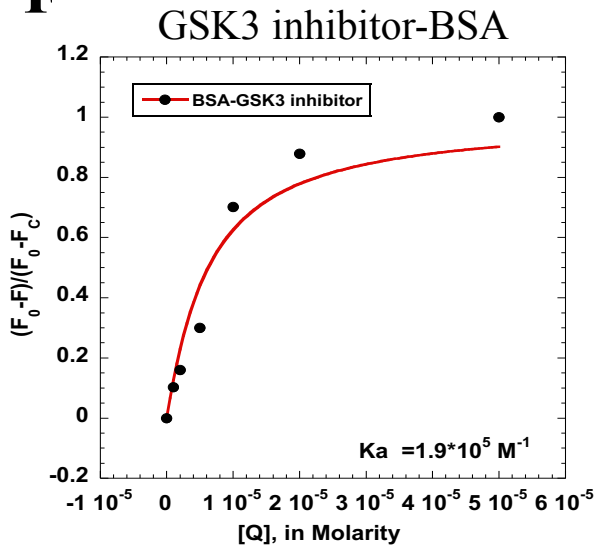

I

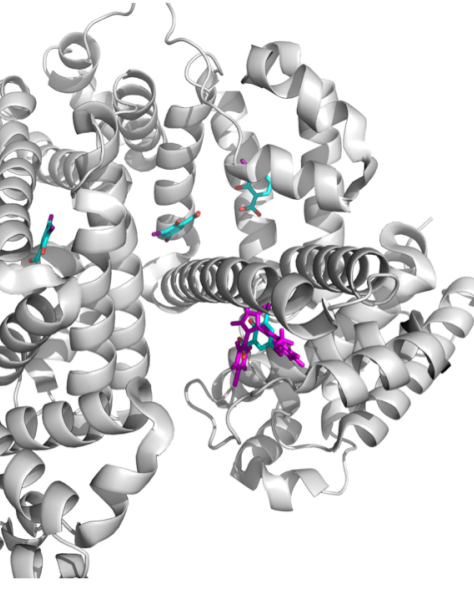

PBS ( 4X EYitakf) under aCC-BYMEM 4. Internationatligense.

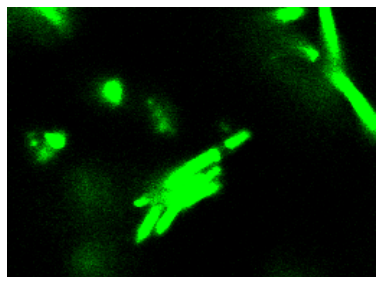

$50 \mathrm{mg} / \mathrm{mL}$ of BSA

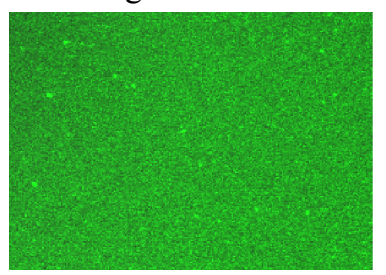

D

Diffusion Coefficients
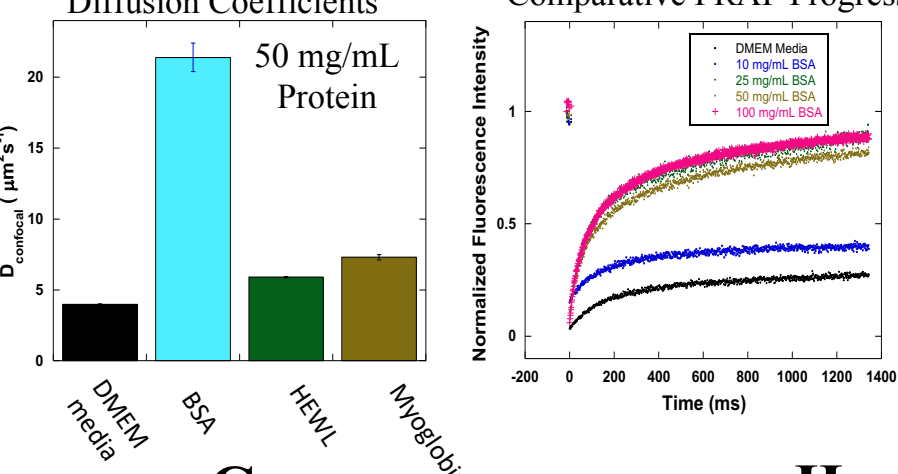

Time (ms)

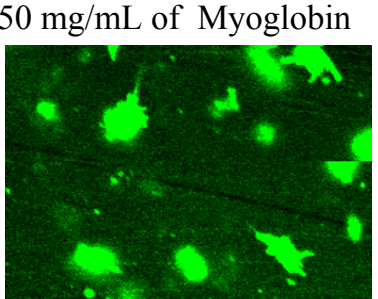

$\mathbf{E}$

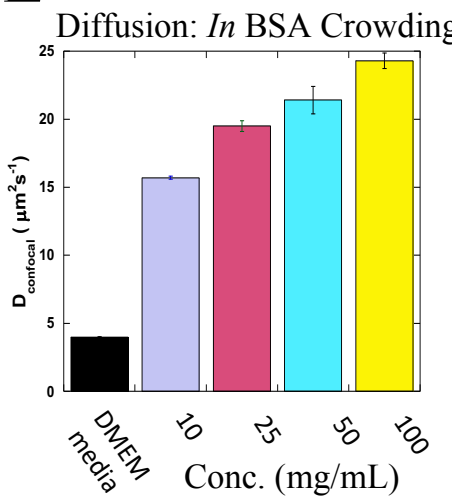

$10 \mathrm{mg} / \mathrm{mL}$ of BSA

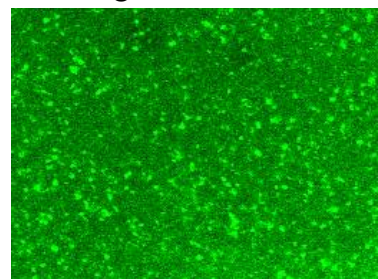

$50 \mathrm{mg} / \mathrm{mL}$ of $\mathrm{HEWL}$

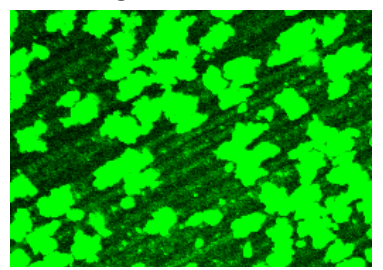

$\mathbf{H}$
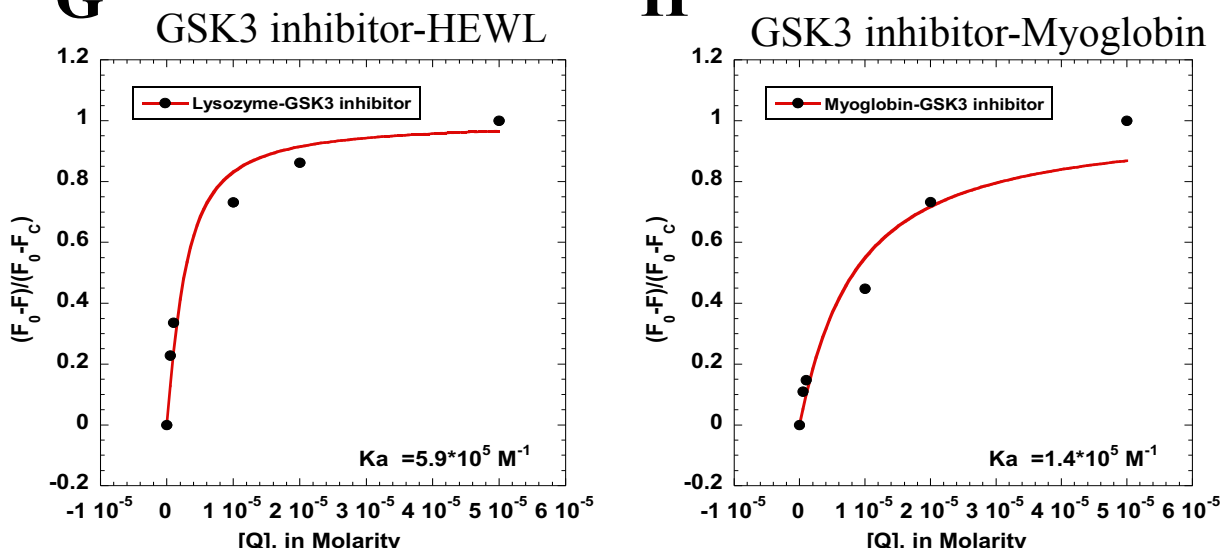

[Q], in Molarity

Figure 6: GSK3 inhibitor in protein crowders: (A) Confocal images of GSK3 inhibitor aggregates in PBS, DMEM media, BSA, HEWL and myoglobin. The disappearance of aggregation in the presence of increasing BSA protein concentrations is also shown. (B) Comparative averaged FRAP profiles $(\mathrm{N}=30 ; \mathrm{R}=0.99$ for each of the fits) and (C) diffusion coefficients in presence of $50 \mathrm{mg} / \mathrm{mL}$ of BSA, HEWL and myoglobin. (D) Comparative averaged FRAP profiles ( $N=30 ; R=0.99$ for each of the fits) and (E) diffusion coefficients of GSK3 inhibitor drug in different amounts of BSA protein. $\left(\mathrm{F}_{0}-\mathrm{F}\right) /\left(\mathrm{F}_{0}-\mathrm{F}_{\mathrm{c}}\right)$ vs $[\mathrm{Q}]$ plots from fluorescence quenching experiments of GSK3 inhibitor-BSA $(\mathrm{R}=0.97) / \mathrm{HEWL}(\mathrm{R}=0.98)$ /myoglobin $(\mathrm{R}=0.98)$ systems in PBS buffer are shown in $(\mathrm{F}-\mathrm{H})$, where $\mathrm{Q}$ is the titrating drug concentration in molarity. (I) BSA (grey) bound to 3,5diiodosalicylic acid (PDB 4JK4, ligand with carbons in cyan). Docking of the GSK3 inhibitor (in pink) to BSA (PDB 4F5S) was performed using different grid centers to capture each of the four binding sites for 3,5diiodosalicylic acid. In one of the binding sites, the GSK3 inhibitor occupied a position similar to that of 3,5-diiodosalicylic acid, showing that the GSK3 inhibitor can bind to a rather buried binding site of BSA. 
(which was not certified by peer review) is the author/funder, who has granted bioRxiv a license to display the preprint in perpetuity. It is m
available underaCC-BY-ND 4.0 International license.

A Quinacrine Diffusion: Additive effects

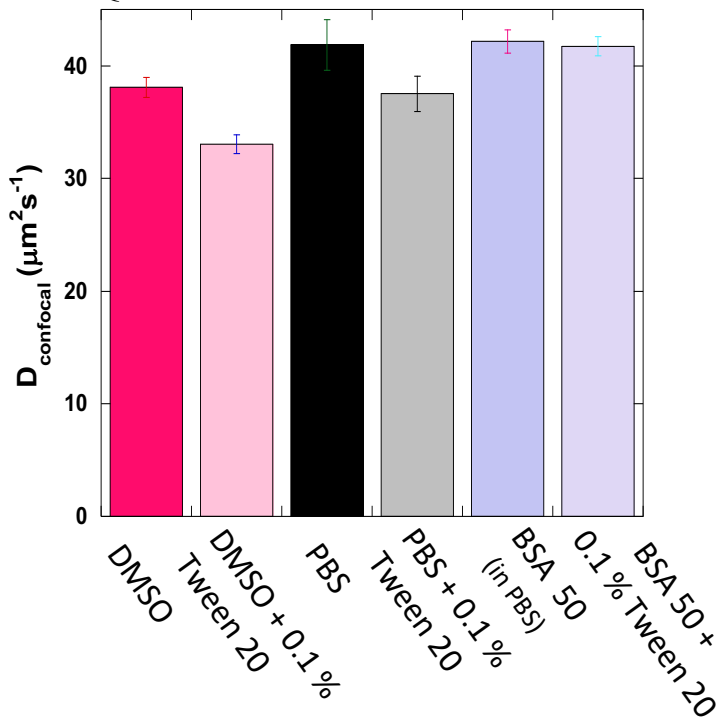

C

Comparative FRAP Progress Curve

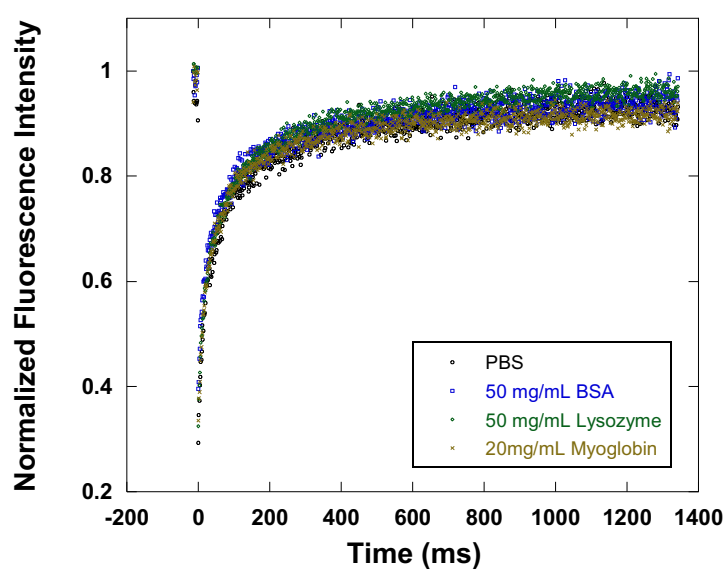

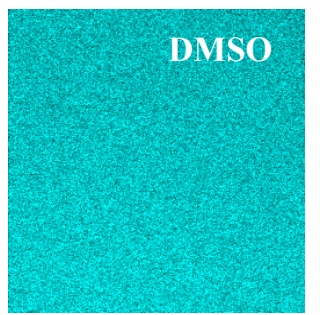

DMSO + Tween 20

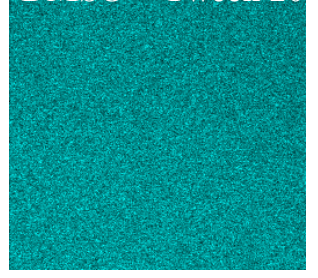

D

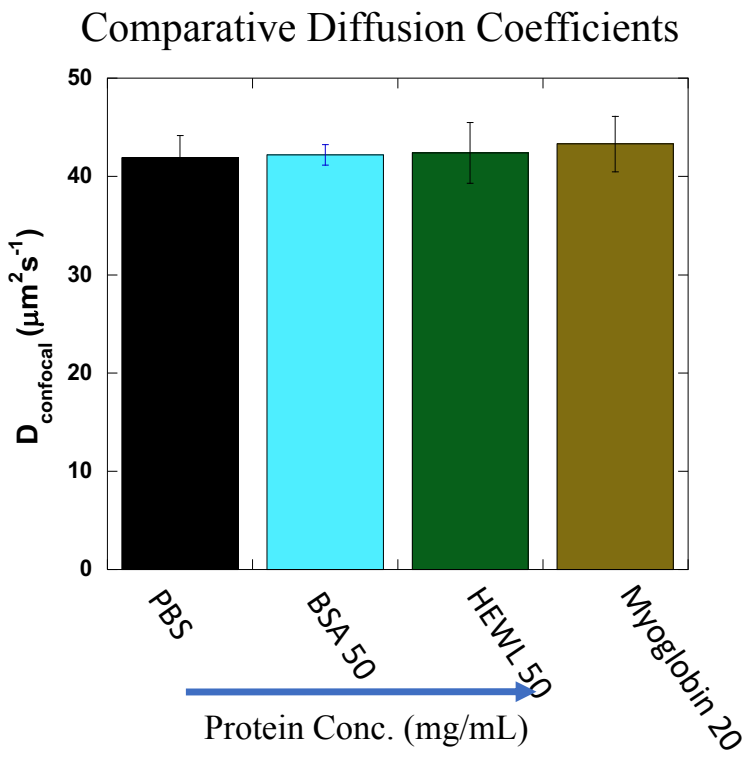

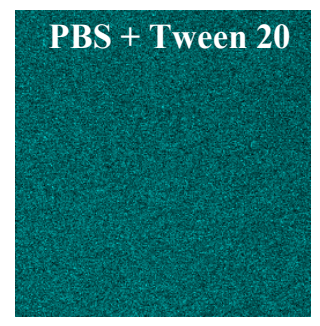

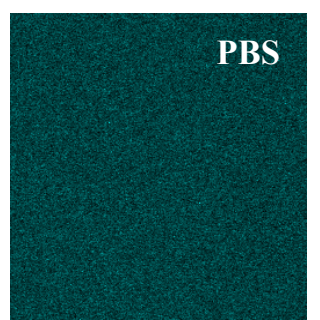

$50 \mathrm{mg} / \mathrm{mL}$ BSA

+ Tween 20

$50 \mathrm{mg} / \mathrm{mL}$ BSA
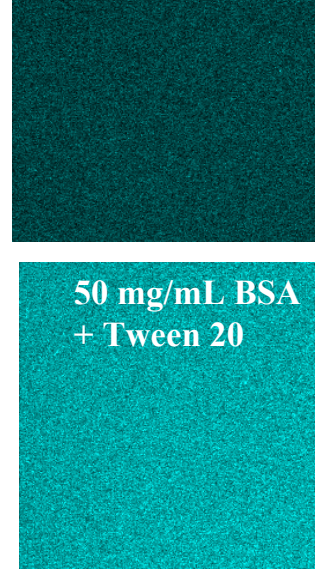

E

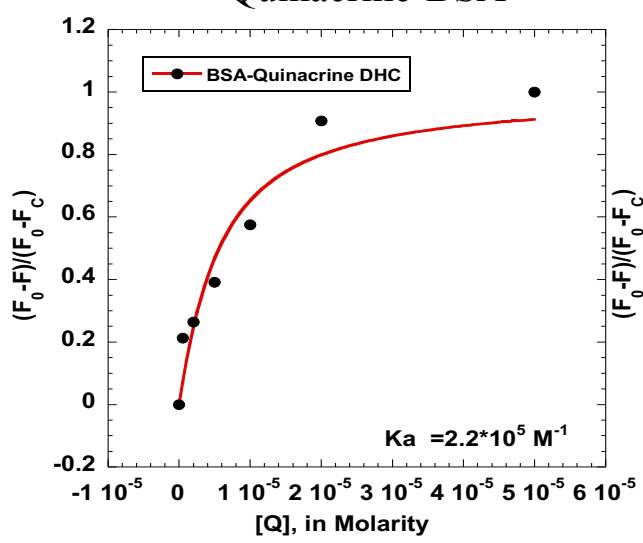

F

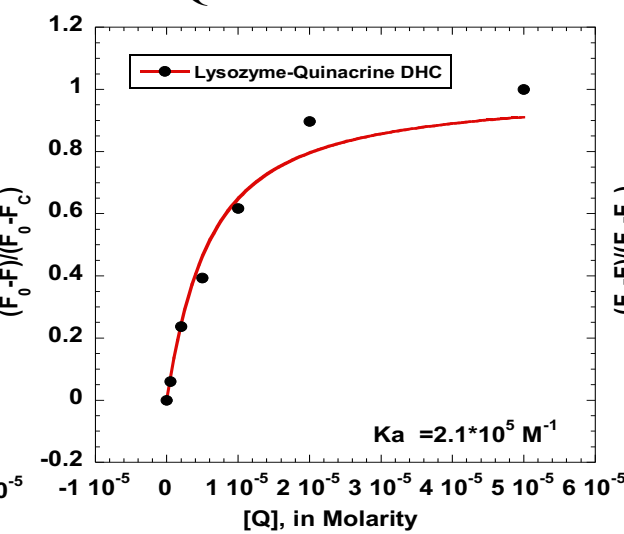

Quinacrine-Myoglobin

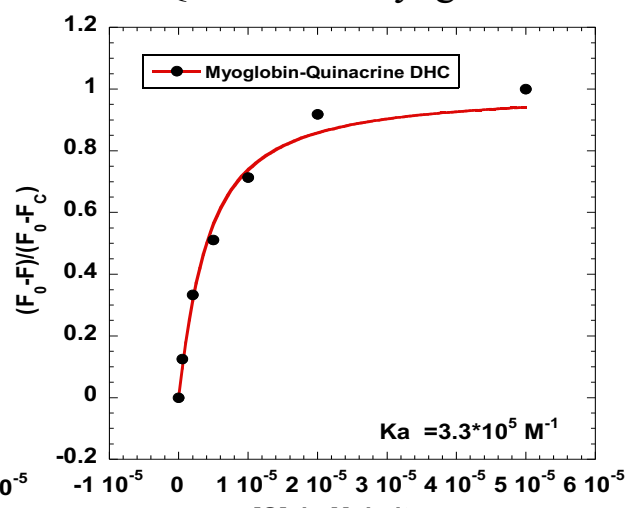

[Q], in Molarity

Figure 7: Quinacrine in protein crowders: Comparative (A) diffusion coefficients and (B) images from confocal microscope of quinacrine DHC in diverse environments with or without tween 20. (C) Comparative averaged FRAP profiles $(\mathrm{R}=0.99$ for each of the fits) and (D) Comparative diffusion coefficients of quinacrine DHC in presence of $50 \mathrm{mg} / \mathrm{mL}$ BSA, $50 \mathrm{mg} / \mathrm{mL}$ HEWL, $20 \mathrm{mg} / \mathrm{mL}$ myoglobin and PBS buffer only. $\left(\mathrm{F}_{0}-\mathrm{F}\right) /\left(\mathrm{F}_{0}-\mathrm{F}\right)$ vs [Q] plots from fluorescence quenching experiments of quinacrine DHC-BSA $(\mathrm{R}=0.97) / \mathrm{HEWL}(\mathrm{R}=0.99) / \mathrm{myoglobin}$ $(\mathrm{R}=0.99)$ system in PBS buffer are shown in $(\mathrm{E}-\mathrm{G})$, where $\mathrm{Q}$ is the titrating drug concentration in $\mathrm{m}$ 


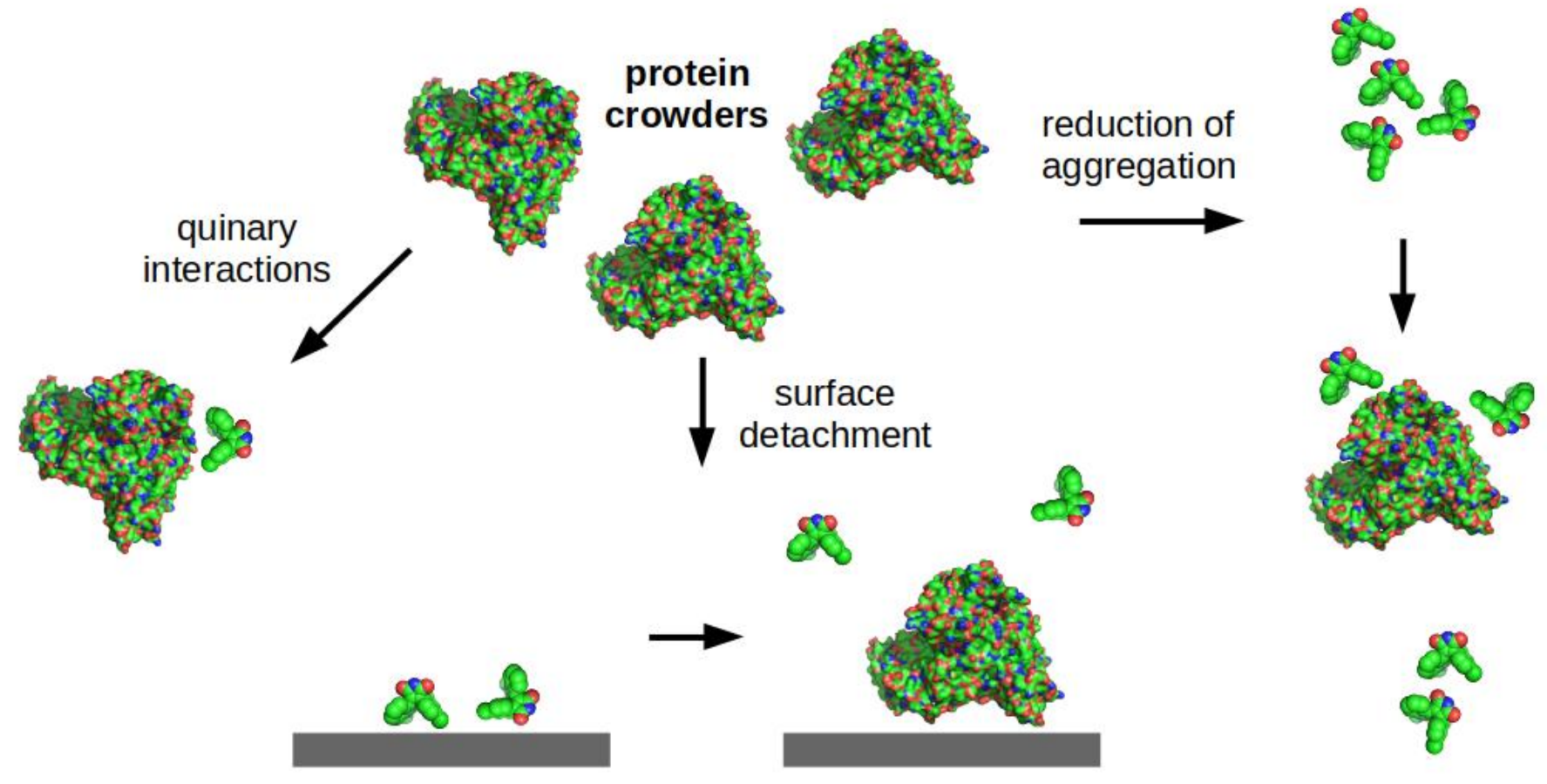

Figure 8: Effects of protein crowders on the translational diffusion coefficients of small molecules: The effects of protein crowders go beyond the slower diffusion due to excluded volume; the diffusion of small molecules can also be slowed down due to quinary interactions, or it can be increased by surface detachment or reduced aggregation of the small molecules. The specific effect of the protein crowder on the translational diffusion coefficient of the small molecule depends on the physico-chemical properties of the crowder and of the small molecule. 